\title{
A taxonomy of fairness and temporal logic problems for Petri nets*
}

\author{
Rodney R. Howell
}

Department of Computing and Information Sciences, Kansas State University, Manhattan, KS 66506, U.S.A.

\section{Louis E. Rosier}

Department of Computer Sciences, The University of Texas at Austin, Austin, TX 78712, U.S.A.

\section{Hs:d-Chun Yen}

Department of Electrical Engineering, National Taiwan University, Tuipei, Taiwan.

Communicated by $\mathbf{H}$. Genrich

Received January 1988

Revised November 1988

\begin{abstract}
Howell, R.R., L.E. Rosier and H.-C. Yen, A taxonomy of fairness and temporal logic problems for Petri nets, Theoretical Computer Science 82 (1991) 341-372.

In this paper, we define a temporal logic for reasoning about Petri nets. We show the model checking problcon for this logic to be PTIME equivalent to the Petri net reachability problem. Using this ir sic and two refinements, we show the fair nontermination problem to be PTIME equivalent to reachability for several definitions of fairness. For other versions of fairness, this problem is shown to be either PTIME equivalent to the boundedness problem or highly undecidable. In all, 24 versions of fairness are examined.
\end{abstract}

\section{Introduction}

In the specification and analysis of concurrent systems, some notion of fairness is often necessary to exclude from consideration certain computations which prevent

* This work was supported in part by U.S. Office of Naval Research Grant No. N00014-86-K-0763 and National Ssience Foundation Grant No. CCR-8711579. A preliminary version was presented at the 13th International Symposium on Mathematical Foundations of Computer Science, Karlovy-Vary (Carlsbad), Czechoslovakia. 
particular events from occurring, even though these events may be possible infinitely often. Many d.finitions of fairness have been proposed (see, e.g., [5-8, 13, 21, 22, 30]), and each has its merit in particular applications. Several versions of fairness have been defined (or adapted) for Petri nets [5-8, 30], a powerful formalism often employed to model concurrent systems [28]. Decidability issues concerning fairness in Petri nets were considered in $[6,7,38]$. One problem examined in these papers is the fair nontermination problem [11]; i.e., for a certain definition of fairness, does there exist an infinite fair computation? Though the decidability of a number of these problems has been determined, only a few rough complexity bounds have been given for those known to be decidable [7]. In [16], we examined the complexity of the fair nontermination problem for conflict-free Petri nets with respect to several definitions of fairness. In this paper, we extend this study to general Petri nets, examining the 24 versions of fairness presented in $[5-8,13,21,22,30]$. The results of this study are summarized in Section 5 (Table 1).

In the study of Petri nets, a scarcity of knowledge concerning computational complexity is by no means unique to the fair nontermination problem. For example, the precise complexity of the reachability problem has remained elusive for many years. For this problem, the most efficient algorithm is not primitive recursive [20,25], whereas the best known lower bound is exponential space [23]. We show in this paper that there is a close relationship between the reachability problem and several of the fair nontermination problems. In particular, we show several of these problems to be equivalent to reachability; i.e., their complexities are equivalent to that of reachability with respect to PTIME many-one reductions. (Throughout this paper, we will use the word "equivalent" in this sense when referring to decision problems.) Thus, to determine the precise complexity of any of these problems (modulo PTIME reductions), it is sufficient to consider only the reachability problem. For examples of other (more classical) problems known to be equivalent to reachability, see [28].

Clearly, since some versions of the fair nontermination problem have been shown to be undecidable $[6,7]$, not all versions are equivalent to reachability. Rather, one of the main points of this paper is that most versions are either highly undecidable (in particular, complete for $\Sigma_{1}^{1}$-the first level of the analytical hierarchy), equivalent to reachability, or equivalent to boundedness (i.e., exponential space complete with respect to PTIME many-one reductions). In some sense, it is easier to show problems to be highly undecidable or equivalent to boundedness than it is to show equivalence to reachability. The reason for this is that highly undecidable problems and exponential space complete problems as a whole are fairly well understood. The reachability problem, however, is not well understood, as is evidenced by the lack of knowledge concerning its complexity. Hence, as a way to overcome this difficulty, we develop in this paper a framework based upon temporal logic for reducing fair nontermination problems to the reachability problem.

For some time, temporal logic has been considered an appropriate formalism for reasoning about systems of concurrent programs [24, 29]. A typical problem involving temporal logic is the model checking problem [10]; i.e., determining whether a 
given structure defines a model of a correctness specification expressed in the temporal logic. The problem can be formally stated in a variety of ways. For the purposes of this paper, model checking is the problem of deciding whether in a given Petri net there is an infinite firing sequence satisfying a given temporal logic formula. This version of model checking (with respect to finite-state structures) was referred to as "determination of truth in a structure" in [33]. What makes the model checking problem useful for us is that most fairness specifications can be stated in some temporal logic (see, e.g., [11]). Thus, a fair nontermination problem can be reduced to a model checking problem. Now in order for this reduction to be useful (for our purposes), the model checking problem must be no harder than reachability. Unfortunately, we were able to show in [16] that for a fairly simple temporal logic, the model checking problem is undecidable, even for conflict-free Petri nets (see also [35]). On the other hand, we were able to show a subset of the logic to be NP-complete for conflict-free nets. This logic utilizes the predicates ge $(p, c)$ (place $p$ is greater than or equal to $c$ ), en $(t)$ (transition $t$ is enabled) and fi(t) (transition $t$ is the next to fire). Call this set of predicates $Q^{\prime}$. The operators used are $F$ (sometime), $\boldsymbol{X}$ (next time), $\wedge$ (and), $\vee$ (or), and $\neg$ (not), where $\neg$ is used only on predicates. The problem with this logic (which we will sall $\tilde{\mathscr{L}}\left(Q^{\prime}, \boldsymbol{F}, \boldsymbol{X}\right)$ ) is that it can only make assertions about finite portions of (possibly infinite) firing sequences. Hence, it is only marginally useful for expressing fairness constraints.

With respect to general Petri nets, it is not hard to see that $\tilde{\mathscr{L}}\left(Q^{\prime}, F, X\right)$ is powerful enough to express reachability. Thus, the model checking problem for this logic is as hard as reachability. In this paper, we extend this logic by adding new predicates which give it the power to specify certain loops. This strategy differs from our analysis in [16], where we kept the set of predicates fixed but restricted the use of the operators in order to develop various logics. With this new logic (which we will call $\tilde{\mathscr{L}}(\boldsymbol{Q}, \boldsymbol{F}, \boldsymbol{X}))$ we associate a variation of the model checking problem which we call the finite model checking problem. This problem is to determine whether there exists a finite firing sequence that satisfies a given formula. The reason we introduce the finite model checking problem is to allow us to use predicates asserting that a firing sequence produces a nonnegative (or zero) net change on a given place; this assertion would not make sense for arbitrary infinite firing sequences. We are then able to show that the finite model checking problem for $\tilde{\mathscr{L}}(\boldsymbol{Q}, \boldsymbol{F}, \boldsymbol{X})$ is equivalent to reachability. In so doing, we develop a methodology for admitting new predicates to the logic without destroying its equivalence to reachability. Because $\tilde{\mathscr{L}}(Q, F, X)$ has the power to specify loops, it can specify that certa: : 'vpes of events occur infinitely often. In particular, suppose we wish to determire whether a Petri net $\mathscr{P}$ is a model for a formula $f \in \mathscr{L}\left(Q^{\prime}, F, X\right)$. We need only to find a finite firing sequence that satisfies $f$ and ends in a loop. We can specify a path of this type in $\tilde{\mathscr{L}}(Q, F, X)$; hence, the model checking problem for $\tilde{\mathscr{L}}\left(Q^{\prime}, F, X\right)$ may be expressed as an instance of the finite model checking problem for $\tilde{\mathscr{L}}(Q, F, X)$. Furthermore, $\tilde{\mathscr{L}}(Q, F, X)$ is powerful enough to express formulas of the form "infinitely often $q$," where $q$ is a Boolean combination of predicates from $Q^{\prime}$. (Call this set of formulas $\mathscr{L}^{x}\left(Q^{\prime}\right)$.) 
Again, we can express the model checking problem for $\mathscr{L}^{\infty}\left(Q^{\prime}\right)$ as an instance of the finite model checking problem for $\tilde{\mathscr{L}}(Q, F, X)$. This answers a question left open in [15] as to whether the model checking problem for $\mathscr{L}^{\infty}\left(Q^{\prime}\right)$ is decidable for conflict-free Petri nets. Perhaps the most important property of $\tilde{\mathscr{L}}(Q, F, X)$ is its ability to succinctly express various definitions of fairness-namely, $\infty$-fairness [5], fdp- $T$ where $T$ is a set of transitions [7], and five types of fairness introduced by Landweber [21] and Carstensen and Valk [8]. As a result, we are able to show the fair nontermination problems for all of these types of fairness to be equivalent to reachability. Thus, $\tilde{\mathscr{L}}(Q, F, X)$ provides a powerful mechanism for showing equivalence of certain fair nontermination problems to reachability. Finally, we might also mention that the existence of such a logic is somewhat surprising in view of the aforementioned negative results of [16].

Since so many of the decidable versions of the fair nontermination problem are equivalent to more classical Petri net problems, one might surmise that the undecidable versions are equivalent to containment and equivalence, which have been shown to be undecidable in [4] and [14], respectively. Since reachability is decidable, it is not hard to see that both of these problems are in $\Pi_{1}$; i.e., they are co-r.e. As Vidal-Naquet [6] and Carstensen [7] have demonstrated, the key step in showing that any version is undecidable is to show that the Petri net under the given fairness constraint can simulate zero-testing. Once zero-testing can be performed, the Petri net can simulate an arbitrary Turing machine. Carstensen [7] has also exhibited another interesting capability of Petri nets under certain fairness constraints: the ability to nondeterministically generate any natural number without running the risk of entering an infinite loop. Thus, unbounded nondeterminism can be simulated. We use this fact to show that these versions are complete for $\Sigma_{1}^{1}$. Hence, these problems are highly undecidable and not equivalent to containment or equivalence. These results may be compared and contrasted with those of $[1-3,9,15,27,34]$.

The remainder of the paper is organized as follows. In Section 2, we give the basic definitions of Petri nets and temporal logic. In Section 3, we develop the logics that are later used to show various types of fair nontermination problems to be equivalent to reachability. In Section 4, we examine the fair nontermination problem for the 24 types of fairness. In most cases, we are able to show the problems to be either highly undecidable, equivalent to reachability, or equivalent to boundedness. One exception is with respect to fairness (as defined in [22]) for bounded Petri nets, as is mentioned in [7]. Although this problem is clearly decidable, we are able to show it to be nonprimitive recursive. We conclude in Section 5 with a summary of our results and a discussion of open problems.

\section{Definitions}

A Petri Net $(\mathrm{PN})$ is a tuple $\left(P, T, \varphi, \mu_{0}\right)$, where $P$ is a finite set of places, $T$ is a finite set of transitions, $\varphi$ is a flow function $\varphi:(P \times T) \cup(T \times P) \rightarrow N$, and $\mu_{0}$ is the 
initial marking $\mu_{0}: P \rightarrow N$, where $N$ is the set of natural numbers. A marking is a mapping $\mu: P \rightarrow N$. We often establish an order on the places, $p_{1}, \ldots, p_{k}$, and designate a marking $\mu$ as a vector in $N^{k}$, where the $i$ th component represents $\mu\left(p_{i}\right)$. A transition $t \in T$ is enabled at a marking $\mu$ iff for every $p \in P, \varphi(p, t) \leqslant \mu(p)$. A transition $t$ may fire at a marking $\mu$ if $t$ is enabled at $\mu$. We then write $\mu \rightarrow \mu^{\prime}$, where $\mu^{\prime}(p)=\mu(p)-\varphi(p, t)+\varphi(t, p)$ for all $p \in P$. A sequence of transitions $\sigma=$ $t_{1} \ldots t_{n}$ is a firing sequence from $\mu_{0}$ iff $\mu_{0} \stackrel{t_{1}}{\rightarrow} \mu_{1} \stackrel{t_{2}}{\rightarrow} \cdots \stackrel{t_{n}}{\rightarrow} \mu_{n}$ for some sequence of markings $\mu_{1}, \ldots, \mu_{n}$. We also write $\mu_{0} \stackrel{\sigma}{\rightarrow} \mu_{n}$, and denote $t_{1} \ldots t_{j}$ by $\sigma[j]$ for $1 \leqslant j \leqslant n$. We extend these notions to infinite firing sequences in the obvious way.

For a $P N \mathscr{P}=\left(P, T, \varphi, \mu_{0}\right)$, the reachability set of $\mathscr{P}$ is the set $R(\mathscr{P})=\left\{\mu \mid \mu_{0} \stackrel{\sigma}{\rightarrow} \mu\right.$ for some $\sigma$ \}. Given a marking $\mu$ of $\mathscr{P}$, the reachability problem (RP) is to determine whether $\mu \in R(\mathscr{P})$. The boundedness problem (BP) is to determine whether $R(\mathscr{P})$ is finite. Throughout this paper, we will define several fairness properties for firing sequences. Given a fairness property $x$, the nontermination problem with respect to $\boldsymbol{x}\left(\mathrm{NTP}^{x}\right)$ is to determine whether there is an infinite firing sequence $\sigma$ in $\mathscr{P}$ that satisfies $x$.

A labeled Petri net is a triple $\mathscr{P}=\left(\mathscr{P}_{1}, \Sigma, h\right)$, where $\mathscr{P}_{1}=\left(P, T, \varphi, \mu_{0}\right)$ is a PN, $\Sigma$ is a finite set of labels, and $h: T \rightarrow \Sigma \cup\{\varepsilon\}$ is a labeling function. We also extend $h: T^{*} \rightarrow \Sigma^{*}$ by $h(\varepsilon)=\varepsilon$ and $h(\sigma t)=h(\sigma) h(t)$. Given a marking $\mu$ of $\mathscr{P}$, we define the terminal language of $\mathscr{P}$ with respect to $\mu$ as $L^{\prime}(\mathscr{P}, \mu)=\left\{h(\sigma) \mid \mu_{0} \stackrel{\sigma}{\rightarrow} \mu\right\}$.

Let $\mathcal{N}$ denote the set of all PNs, $\mathscr{T}^{*}$ denote all finite firing sequences of nets in $\mathcal{N}, \mathscr{T}^{\omega}$ denote all infinite firing sequences of nets in $\mathcal{N}$, and $\mathscr{T}^{\infty}=\mathscr{T}^{*} \cup \mathscr{T}^{\omega}$. A predicate is a partial function $q: \mathcal{N} \times \mathscr{T}^{\infty} \times N \rightarrow$ true, false\}. A well-formed formula (wff) is either a predicate or of the form $\neg f, f \wedge g, X f$, or $f U g$, where $f$ and $g$ are wffs. We will use the notation $\langle\mathscr{P}, \sigma, n\rangle \models f$ to signify that the wff $f$ holds after $n$ transitions have fired in the firing sequence $\sigma$ of the PN $\mathscr{P}$. More formally, for $\mathscr{P} \in \mathcal{N}, \sigma$ a (finite or infinite) firing sequence of $\mathscr{P}, n \in N$, and a predicate $q$, we say $\langle\mathscr{P}, \sigma, n\rangle \models q$ iff $q(\mathscr{P}, \sigma, n)=$ true. For a firing sequence $\sigma$ of $\mathscr{P}$ and wffs $f$ and $g$, we say.

- $\langle\mathscr{P}, \sigma, n\rangle \vDash \neg f$ iff not $(\langle\mathscr{P}, \sigma, n\rangle \vDash f)$;

- $\langle\mathscr{P}, \sigma, n\rangle \vDash X f$ iff $\langle\mathscr{P}, \sigma, n+1\rangle \vDash f$;

- $\langle\mathscr{P}, \sigma, n\rangle \vDash f U g$ iff $\exists r\rangle n$ such that $\langle\mathscr{P}, \sigma, r\rangle \vDash g$ and $\forall s, n<s\langle r,\langle\mathscr{P}, \sigma, s\rangle \vDash f$;

- $\langle\mathscr{P}, \sigma, n\rangle \vDash f \wedge g$ iff $\langle\mathscr{P}, \sigma, n\rangle \vDash f$ and $\langle\mathscr{P}, \sigma, n\rangle \vDash g$.

We also use the following abbreviations:

- $f \vee g \equiv \neg(\neg f \wedge \neg g)$;

- $f \supset g \equiv \neg f \vee g$;

- $F f \equiv$ true $U f$

- $\boldsymbol{G} \boldsymbol{f} \equiv \neg \boldsymbol{F} \neg f$.

We say that $\mathscr{P}$ is a ( inite) model for $f$ iff there is an infinite (finite, respectively) firing sequence $\sigma$ in $\mathscr{P}$ such that $\langle\mathscr{P}, \sigma, 0\rangle \models f$. Let $\mathscr{P}$ be a set of wffs. The (finite) model checking problem with respect to $\mathscr{F}$, denoted $\mathrm{MCP}(\mathscr{F})(\mathrm{FMCP}(\mathscr{F})$, respectively), is to determine whether a given $P N \mathscr{P}$ is a (finite) model for a given formula $f \in \mathscr{F}$. Let $Q$ be a set of predicates. We then define - $\mathscr{L}(Q)=\{f \mid f$ is a wff using predicates from $Q\}$ 
- $\tilde{\mathscr{L}}(Q, F, \boldsymbol{X})=\{f \mid f$ is a wff using predicates from $Q$ and the operators $F, \boldsymbol{X}, \wedge$, $\checkmark$, and $\neg$, such that $\neg$ is used only on predicates $\}$; and

- $\mathscr{L}^{x}(Q)=\{G F f \mid f$ is a Boolean combination of predicates from $Q\}$.

For a $P N \mathscr{P}=\left(P, T, \varphi, \mu_{0}\right)$, a wff $f$, and a natural number $n$, we define the model language of $\mathscr{P}$ with respect to $f$ and $n$ as $L^{m}(\mathscr{P}, f, n)=\{\sigma \mid \sigma$ is finite and $\langle\mathscr{P}, \sigma, n\rangle \vDash f\}$. A set $\mathscr{F}$ of wffs is said to be $R P$-decidable iff for all $f \in \mathscr{F}, \mathscr{P} \in \mathcal{N}$, we can construct in PTIME a labeled PN $\mathscr{P}^{\prime}=\left(\mathscr{P}_{1}^{\prime}, \Sigma, h\right), \mathscr{P}_{1}^{\prime}=\left(P^{\prime}, T^{\prime}, \varphi^{\prime}, \mu_{0}^{\prime}\right), P^{\prime}=p_{1}, \ldots, p_{k}$, and a marking $\mu^{\prime}$ on $p_{1}, \ldots, p_{k-1}$ such that for all $n \in N, L^{m}(\mathscr{P}, f, n)=L^{\prime}\left(\mathscr{P}^{\prime},\left(\mu^{\prime}, n\right)\right)$. We will refer to $p_{k}$ as the marker.

\section{A temporal logic for Petri nets}

In this section, we will present a temporal logic for reasoning aiout Petri nets such that if $\mathscr{F}$ is the set of all wffs in the logic, then $\operatorname{FMCP}(\mathscr{F}) \equiv_{\text {PTIME }}$ RP. The logic will be $\tilde{\mathscr{L}}(Q, F, \boldsymbol{X})$ for a set $Q$ of predicates to be defined later. Even though $\operatorname{FMCP}(\tilde{\mathscr{L}}(Q, F, X))$ is no harder than $R P, Q$ will contain a sufficient variety of predicates to provide a powerful mechanism for showing fair nontermination problems to be equivalent to RP. Furthermore, certain restrictions of the logic provide interesting extensions to the results shown in [16]. The first extension we show is that $\operatorname{MCP}\left(\tilde{\mathscr{L}}\left(Q^{\prime}, F, X\right)\right) \equiv_{\text {PTIME }} R P$, where $Q^{\prime}$ is the set of predicates from the logic developed in [16]. The second extension is that $\operatorname{MCP}\left(\mathscr{L}^{\infty}\left(Q^{\prime}\right)\right) \equiv_{\text {PTIME }} \operatorname{RP}$. Both of these extensions may be considered refinements of the main result of this sectionthat $\operatorname{FMCP}(\tilde{\mathscr{L}}(Q, F, X)) \equiv_{\text {PTIME }}$ RP. All of these logics will be used in the next section to show various fair nontermination problems to be equivalent to RP. The main result of this section may therefore be viewed as an umbrella under which a number of the subsequent results in this paper are derived.

In order to show that $\operatorname{FMCP}(\tilde{\mathscr{L}}(Q, F, X)) \leqslant_{\text {PTIME }} R P$, we will first show that the reduction holds for any $R P$-decidable set of predicates whose negations are also RP-decidable. We will then define $Q$ and show that both $Q$ and $\tilde{Q}=\{\neg q \mid q \in Q\}$ are RP-decidable. We first state the following lemma, which follows immediately from the definition of the terminal language of a labeled PN.

Lemma 3.1. Given a labeled $P N \mathscr{P}=\left(\mathscr{P}_{1}, \Sigma, h\right)$ and a marking $\mu, L^{\prime}(\mathscr{P}, \mu) \neq \emptyset$ iff $\mu \in \boldsymbol{R}\left(\mathscr{P}_{1}\right)$.

We can now give the following lemma, relating the FMCP to the RP.

Lemma 3.2. For a set of wffs $\mathscr{F}$, if $\mathscr{F}$ is RP-decidable, then $\mathrm{FMCP}(\mathscr{F}) \leqslant_{\text {PT!ME }} \mathrm{RP}$.

Proof. Given an RP-decidable set $\mathscr{F}$ of wffs, let $f \in \mathscr{F}$, and let $\mathscr{P}$ be an arbitrary PN. Since $\mathscr{F}$ is RP-decidable, we can construct in PTIME a labeled PN $\mathscr{P}^{\prime}=$ 
$\left(\mathscr{P}_{1}^{\prime}, \Sigma, h\right)$ and a marking $\mu$ such that $L^{\prime}\left(\mathscr{P}^{\prime}, \mu\right)=L^{m}(\mathscr{P}, f, 0)$. Then $\mathscr{P}$ is a finite model for $f$ iff $L^{m}(\mathscr{P}, f, 0) \neq \emptyset$ iff $L^{\prime}\left(\mathscr{P}^{\prime}, \mu\right) \neq \emptyset$ iff $\mu \in R\left(\mathscr{P}_{1}^{\prime}\right)$. Therefore, $\operatorname{FMCP}(\mathscr{F}) \leqslant_{\text {PTIME }}$ RP.

The following theorem now gives a framework for defining our set of predicates.

Theorem 3.3. If a set of predicates $Q$ is $R P$-decidable and $\tilde{Q}=\{\neg q \mid q \in Q\}$ is $R P$ decidable, then $\operatorname{FMCP}(\tilde{\mathscr{L}}(Q, F, X)) \leqslant_{\text {PTIME }} \mathrm{RP}$.

Proof. We will show by induction on the structure of $f \in \tilde{\mathscr{L}}(Q, F, X)$ that for any PN $\mathscr{P}$, we can construct in PTIME a labeled PN $\mathscr{P}^{\prime}$ and a submarking $\mu$ such that for all $n \in N, L^{\prime}\left(\mathscr{P}^{\prime},(\mu, n)\right)=L^{m}(\mathscr{P}, f, n)$. The theorem will then follow from Lemma 3.2. If $f \in Q \cup \tilde{Q}$, this is trivial. Therefore, assume we have $f_{1}, f_{2} \in \tilde{\mathscr{L}}(Q, F, X)$ such that for any PN $\mathscr{P}$, we can construct in PTIME labeled PNs $\mathscr{P}_{1}^{\prime}, \mathscr{P}_{2}^{\prime}$ and markings $\mu_{1}, \mu_{2}$ such that for all $n \in N, L^{\prime}\left(\mathscr{P}_{1}^{\prime},\left(\mu_{1}, n\right)\right)=L^{m}\left(\mathscr{P}, f_{1}, n\right)$ and $L^{\prime}\left(\mathscr{P}_{2}^{\prime},\left(\mu_{2}, n\right)\right)=$ $L^{m}\left(\mathscr{P}, f_{2}, n\right)$.

Case 1: $f=f_{1} \wedge f_{2}$. We construct $\mathscr{P}^{\prime}$ from $\mathscr{P}_{1}^{\prime}$ and $\mathscr{P}_{2}^{\prime}$ as follows. Let $T_{1 a}\left(T_{2 a}\right)$ be the set of all transitions labeled $a$ in $\mathscr{P}_{1}^{\prime}\left(\mathscr{P}_{2}^{\prime}\right.$, respectively) for all $a \in \Sigma \cup\{\varepsilon\}$, where $\Sigma=\left\{a_{1}, \ldots, a_{m}\right\}$ is the 'lphabet of $L^{m}(\mathscr{P}, f, n)$. We relabel all transitions in $\mathscr{P}_{1}^{\prime}$ and $\mathscr{P}_{2}^{\prime}$ as $\varepsilon$, and add $m+1$ new transitions and $2 m+3$ new places, as is shown in Figure 1. We define $\mu$ as:

- $\mu\left(p_{i j}\right)=\mu_{i}\left(p_{i j}\right)$ for $i=1,2, j=1, \ldots, k_{i}-1$;

- $\mu\left(p_{i k_{i}}\right)=0$ where $p_{i k_{i}}$ is the marker for $\mathscr{P}_{i}^{\prime}, i=1,2$;

- $\mu\left(\mathrm{ack}_{i}\right)=1$ for $i=1,2$;

- $\mu\left(\mathrm{en}_{i j}\right)=0$ for $i=1,2, j=1, \ldots, m$.

$p_{\text {mark }}$ is the marker for $\mathscr{P}^{\prime}$. It is not hard to see that for all $n \in N, \sigma \in L^{\prime}\left(\mathscr{P}^{\prime},(\mu, n)\right)$ iff $\sigma \in L^{\prime}\left(\mathscr{P}_{1}^{\prime},\left(\mu_{1}, n\right)\right) \cap L^{\prime}\left(\mathscr{P}_{2}^{\prime},\left(\mu_{2}, n\right)\right) \quad$ iff $\quad \sigma \in L^{m}\left(\mathscr{P}, f_{1}, n\right) \cap L^{m}\left(\mathscr{P}^{\prime}, f_{2}, n\right) \quad$ iff $\langle\mathscr{P}, \sigma, n\rangle \models f_{1} \wedge f_{2}$ iff $\sigma \in L^{m}(\mathscr{P}, f, n)$.

Case 2: $f=f_{1} \vee f_{2} . \mathscr{P}^{\prime}$ nondeterministically chooses to simulate sne of $\mathscr{P}_{1}^{\prime}$ or $\mathscr{P}_{2}^{\prime}$; the other, it brings immediately to its final marking with a zero marker value. $\mathscr{P}^{\prime}$ can also transfer all tokens from the markers of $\mathscr{P}_{1}^{\prime}$ and $\mathscr{P} ! 2$ to its marker. The details is: ieft to the reader.

Case 3: $f=\boldsymbol{X} f_{1} . \mathscr{P}^{\prime}$ simulates $\mathscr{P}_{1}^{\prime}$, but in order to reach its final marking, it must subtract 1 from the marker.

Case 4: $f=F f_{1}$. This is the same as Case 3 except that $\mathscr{P}^{\prime}$ can subtract more than 1 from the marker.

We are now ready to define our predicates. For a $\mathrm{PN} \mathscr{P}=\left(P, T, \varphi, \mu_{0}\right), p \in P$, $t \in T, c, n \in N$, and a finite firing sequence $\sigma$, let:

- $\langle\mathscr{P}, \sigma, n\rangle \models \operatorname{ge}(p, c)$ iff $\mu_{0} \stackrel{\sigma[n]}{\longrightarrow} \mu$ and $\mu(p) \geqslant c$;

- $\langle\mathscr{P}, \sigma, n\rangle \models f(t)$ iff $t$ is the $(n+1)$ st transition in $\sigma$; 


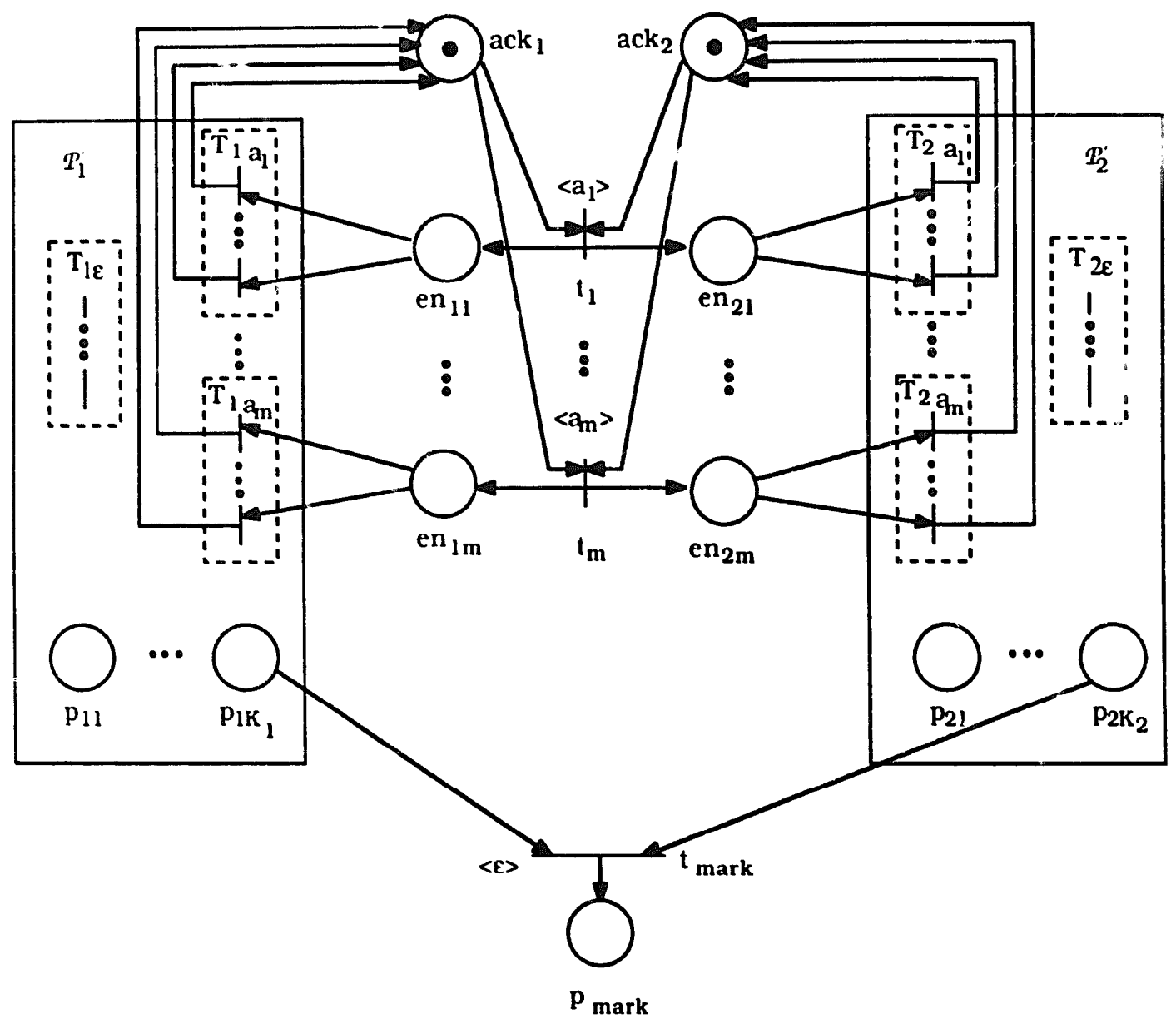

Fig. 1. $\mathscr{P}^{\prime}$ for $f_{1} \wedge f_{2}$.

- $\langle\mathscr{P}, \sigma, n\rangle \vDash \operatorname{lp}(p)$ iff $\mu_{0} \stackrel{\sigma[n]}{\longrightarrow} \mu_{1}, \mu_{0} \stackrel{G}{\rightarrow} \mu_{2}$, and $\mu_{1}(p) \leqslant \mu_{2}(p)$;

- $\langle\mathscr{P}, \sigma, n\rangle \vDash \operatorname{zl}(p)$ iff $\mu_{0} \stackrel{\sigma[n]}{\longrightarrow} \mu_{1}, \mu_{0} \stackrel{\sigma}{\rightarrow} \mu_{2}$, a ald $\mu_{1}(p)=\mu_{2}(p)$;

- $\langle\mathscr{P}, \sigma, n) \vDash \operatorname{co}(\mu)$ iff $\mu_{0} \stackrel{\text { or }[n]}{\longrightarrow} \mu_{1}$ and there is a $\mu_{2} \in R\left(P, T, \varphi, \mu_{1}\right)$ such that $\mu_{2} \geqslant \mu$. Let $Q$ be the set of all of the above predicates for all places $p$ and transitions $t$, and $\tilde{Q}=\{q \mid \neg q \in Q\}$. We wish to show that $\operatorname{FMCP}(\tilde{\mathscr{L}}(Q, F, X)) \leqslant_{\text {PTIME }} \operatorname{RP}$. From Theorem 3.3, we need only show that $Q$ and $\tilde{Q}$ are RP-decidable. For most tements of $Q \cup \tilde{Q}$, this is straightforward. The n!ain difficulty lies with $\neg \operatorname{co}(\mu)$. In showing $\neg \operatorname{co}(\mu)$ to be RP-decidable, we will construct a PN that will in some sense produce all markings from which no marking greater than or equal to $\mu$ can be reached. In order to construct such a PN, we first construct a modified Turing machine (TM) that accomplishes the same purpose. This modified TM will be such that using Lipton's construction [23], we can transform it to a PN. In order to define the precise function of the modified TM, we introduce the following notation. Given an unmarked $P N \mathscr{P}=(P, T\urcorner$,$) , a marking \mu$, and a positive $c$, let $S(\mathscr{P}, \mu)=$ $\left\{\mu_{0} \mid \forall \mu^{\prime} \geqslant \mu, \quad \mu^{\prime} \notin R\left(P, T, \varphi, \mu_{0}\right)\right\}, \quad$ and $\quad S^{\prime}(\mathscr{P}, \mu, c)=\left\{\mu_{0} \#^{2^{(n n \log n]}} \mid \mu_{0} \in S(\mathscr{P}, \mu)\right\}$, where $n$ is the number of bits needed to encode $\mathscr{P}$ and $\mu$. Also, let $\bar{S}(\mathscr{P}, \mu)$ and $\bar{S}^{\prime}(\mathscr{P}, \mu, c)$ denote the complements of $S(\mathscr{P}, \mu)$ and $S^{\prime}(\mathscr{P}, \mu, c)$, respectively. We 
now give two lemmas which define the function of the modified TM and give its construction.

Lemnid 3.4. There is a positive constant c such that for any unmarked $P N \mathscr{P}=(P, T, \varphi)$ and marking $\mu$, we can construct in PTIME a linearly bounded automaton (LBA) $M$ that accepts the set $S^{\prime}(\mathscr{P}, \mu, c)$.

Proof. For any fixed constant $c$, we can clearly verify deterministically in linear space whether the input is syntactically correct and the number of \#s is correct. From [31], if $\mu_{0} \stackrel{\sigma_{1}}{\rightarrow} \mu_{1} \geqslant \mu$, then $\mu_{0} \stackrel{\sigma_{2}}{\rightarrow} \mu_{2} \geqslant \mu$ such that the length of $\sigma_{2}$ is no more

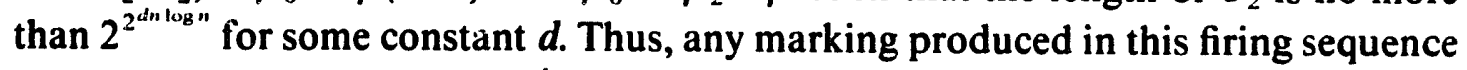
can be stored in $\operatorname{size}\left(\mu_{0}\right)+2^{c n \log n}$ bits for some constant $c . \bar{S}^{\prime}(\mathscr{P}, \mu, c)$ is t'verefore accepted by an LBA $M^{\prime}$ that can be constructed in PTIME. From $[18,36]$, there is \&n LBA $M$ that accepts $S^{\prime}(\mathscr{P}, \mu, c)$. An inspection of the proofs in $[18,36]$ reved that $M$ can be constructed from $M^{\prime}$ in PTIME.

Lemma 3.5. Let $\mathscr{P}, \mu$, and $n$ be as in Lemma 3.4, and let $k$ be the number of places in 5 . We can construct in PTIME an $\mathrm{O}\left(2^{a n \log n}\right)$ space bounded TM M with no input tape, augmented with $k$ unbounded increment-only counters such that the set of final counter values in all accepting computations is $S(\mathscr{P}, \mu)$.

Proof. Let $d$ be the constant from [31] mentioned in the proof of Lemma 3.4. We define a function $g$ mapping markings to markings such that

$$
g(\mu)(p)= \begin{cases}\mu(p) & \text { if } \mu(p) \leqslant 2^{2^{d n|\log n|}} \\ 2^{2^{d n|\log n|}} & \text { otherwise. }\end{cases}
$$

It follows from [31] that $\mu_{0} \in S(\mathscr{P}, \mu)$ iff $g\left(\mu_{0}\right) \in S(\mathscr{P}, \mu)$. We can therefore construct $M$ to operate as follows. $M$ first nondeterministically generates $k$ nonnegative integers no larger than $2^{2^{\ln _{\text {llog }} 1}}$ and stores them on its worktape and on the $k$ counters. If any of these values are exactly $2^{2^{\tan \log n 1}}$, the corresponding counters may be incremented arbitrarily many times. $M$ then writes $\#^{2^{(n|\log n|}}$ on its worktape, where $c$ is the constant from Lemma 3.4, and simulates the machine given by Lemma 3.4 on the contents of its worktape. The result follows from Lemma 3.4 .

We are now ready to show the main result of this section, that $\operatorname{FMCP}(\tilde{\mathscr{L}}(Q, F, X))$ is equivalent to RP. From this result we will subsequently derive two refinements conce: ning logics developed in [16]; these refinements will be given in Theorems 3.7 and 3.9. We will then use Theorem 3.6 and its refinements in Section 4 to show seven fair nontermination problems to be equivalent to RP. The reason we can use a finite model checking problem to encode a fair nontermination problem is that $\tilde{\mathscr{L}}(Q, F, \boldsymbol{X})$ has the power to express certain loops which may be iterated to produce an infinite "fair" path. Thus, Theorem 3.6 is an umbrella under which powerful 
machinery is developed for proving certain fair nonterminstion problems to be equivalent to RP.

Theorem 3.6. $\operatorname{FMCP}(\tilde{\mathscr{L}}(Q, F, X)) \equiv_{\mathrm{PTIME}} \mathrm{RP}$.

Proof. Let $\mathscr{P}=\left(P, T, \varphi, \mu_{0}\right)$ be an arbitrary $\mathrm{PN}$, and let $\mu$ be an arbitrary marking of $\mathscr{P}$. Clearly, $\mu \in R(\mathscr{P})$ iff there is a finite firing sequence $\sigma$ such that

$$
\begin{aligned}
\langle\mathscr{P}, \sigma, 0\rangle & \vDash\left[\bigwedge_{p \in P}(\operatorname{ge}(p, \mu(p)) \wedge \neg \operatorname{ge}(p, \mu(p)+1))\right] \\
\vee & {\left[\bigwedge_{p \in P}(\operatorname{ge}(p, \mu(p)) \wedge \neg \operatorname{ge}(p, \mu(p)+1))\right] . }
\end{aligned}
$$

Thus, RP $\leqslant_{\text {PTIME }} \operatorname{FMCP}(\tilde{\mathscr{L}}(Q, F, X))$.

In order to show that FMCP $(\tilde{\mathscr{L}}(Q, F, X)) \leqslant_{\text {PTIME }} R P$, we need only to show that $Q$ and $\tilde{Q}$ are RP-decidable; the theorem then follows from Theorem 3.3. Let $\mathscr{P}=\left(P, T, \varphi, \mu_{0}\right)$ be an arbitrary PN. We need to show that for any element $q$ of $Q \cup \tilde{Q}$, we can construct in PTIME a labeled PN $\mathscr{P}^{\prime}$ and a marking $\mu^{\prime}$ such that for any $n \in N, L^{m}(\mathscr{P}, q, n)=L^{\prime}\left(\mathscr{P}^{\prime},\left(\mu^{\prime}, n\right)\right)$. Since most of the cases are straightforward, we will only show the cases $\operatorname{ge}\left(p_{i}, c\right)$ and $\neg \operatorname{co}(\mu)$.

Case 1: $q=\operatorname{ge}\left(p_{i}, c\right)$. We construct $\mathscr{P}^{\prime}$ as shown in Fig. 2. Let $\mu^{\prime}\left(\mathrm{en}_{2}\right)=1, \mu^{\prime}(p)=0$ if $p \neq e_{2}$ and $p \neq p_{\text {mark }}$, where $p_{\text {mark }}$ is the marker. In order to reach $\left(\mu^{\prime}, n\right), \mathscr{P}^{\prime}$ must pass through two phases. In the first phase, $\mathscr{P}^{\prime}$ simulates $n$ transitions of a firing sequence $\sigma$ on two copies of $\mathscr{P}$. In the second phase, the remainder of $\sigma$ is simulated on one of the copies. At any time, the transitions $t_{z_{i}}, \ldots, t_{z_{h}}, t_{z_{1}}^{\prime}, \ldots, t_{z_{k}}^{\prime}$ may fire. These transitions enable no new transitions, but allow all places in both copies of $\mathscr{P}$ to be brought to after the simulation of $\sigma$. Furthermore, $t_{\mathrm{ge}}$ must fire exactly once, guaranteeing that $p_{i}$ is at least $c$ after $\sigma[n]$ is executed in $\mathscr{P}$. It can therefore be seen that $L^{\prime \prime}(\mathscr{P}, q, n)=L^{\prime}\left(\mathscr{P}^{\prime},\left(\mu^{\prime}, n\right)\right)$.

Case 2: $q=\neg \operatorname{co}(\mu)$. Before we construct $\mathscr{P}^{\prime}$, we wiii construct a PN $\mathscr{P}^{\prime \prime}$ with designated piaces $p_{1}^{\prime \prime}, \ldots, p_{k}^{\prime \prime}$, where $k$ is the number of places in $\mathscr{P}$, and a submarking $\mu^{\prime \prime}$ on the remaining places in $\mathscr{P}^{\prime \prime}$, such that for any marking $\mu_{1}$ of $\mathscr{P},\left(\mu_{1}, \mu^{\prime \prime}\right) \in R\left(\mathscr{P}^{\prime \prime}\right)$ iff $\mu_{1} \in S((P, T, \varphi), \mu)$. Let $M$ be the machine given by Lemma 3.5 to compute $S((P, T, \varphi), \mu)$. Since the worktape of $M$ is bounded by $O\left(2^{a n l o g n}\right)$ space, it can be simulated by four $\mathrm{O}\left(2^{2^{4 n+1 k n}}\right)$ bounded counters; furthermore, this construction can be done in PTIME. From [23], we can construct in PTIME a PN and a marking $\mu$ " such that any firing sequence yielding $\mu^{\prime \prime}$ simulates an accepting computation of $M$ without its $k$ unbounded counters. Since the $k$ unbounded counters are increment-only, they can clearly be implemented by $k$ places in the PN. We have therefore constructed $\mathscr{P}^{\prime \prime}$ and $\mu^{\prime \prime}$. The construction of $\mathscr{P}^{\prime}$ and $\mu^{\prime}$ from $\mathscr{P}, \mathscr{P}^{\prime \prime}$, and $\mu^{\prime \prime}$ is now similar to Case 1 and is shown in Fig. 3. It should be clear that $L^{\prime \prime}(\mathscr{P}, q, n)=L^{\prime}\left(\mathscr{P}^{\prime},\left(\mu^{\prime}, n\right)\right)$. 


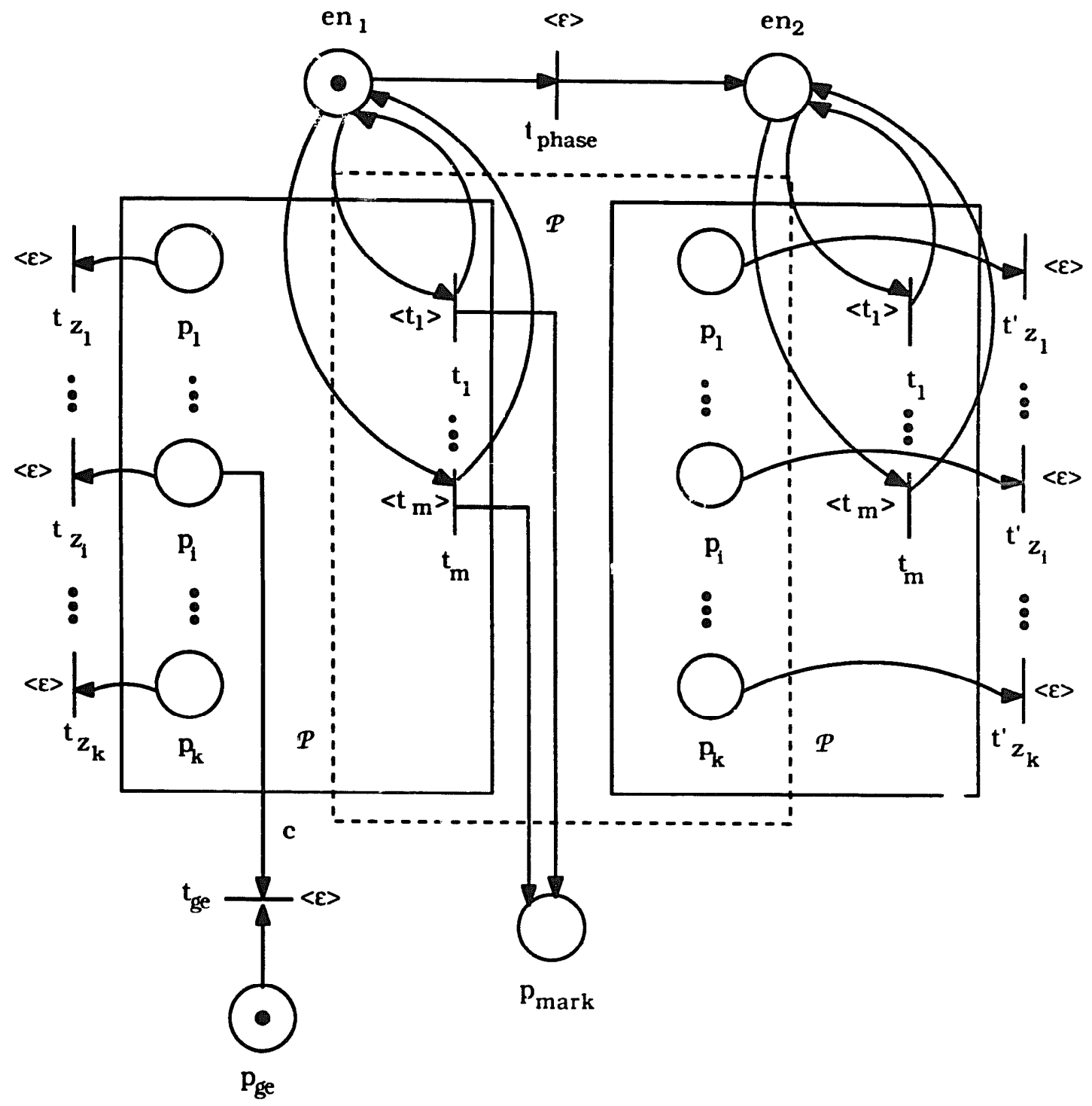

Fig. 2. $\not p^{\prime}$ for $\operatorname{ge}\left(p_{i}, c\right)$.

We now will examine two logics developed in [16]. We can show that the MCP for both problems can be expressed as restrictions of $\operatorname{FMCP}(\tilde{\mathscr{L}}(Q, F, X))$. We will use these two logics in Theorems 4.2 and 4.10 to give succinct reductions from various fair nontermiration problems to RP.

Let $Q^{\prime}$ be the set of predicates ge $(p, c)$ and $f(t)$ extended to infinite firing sequences, and let $\tilde{Q}^{\prime}=\left\{\neg q \mid q \in Q^{\prime}\right\}$. $\operatorname{MCP}\left(\tilde{\mathscr{L}}\left(Q^{\prime}, F, X\right)\right)$ was shown in [16] to be NP-complete for conflict-free PNs. (The logic in [16] also included predicates asserting that a transition $t$ is enabled; this assertion and its negation can clearly be encoded in $\tilde{\mathscr{L}}\left(Q^{\prime}, \boldsymbol{F}, \boldsymbol{X}\right)$.) Although $\tilde{\mathscr{L}}\left(Q^{\prime}, \boldsymbol{F}, \boldsymbol{X}\right)$ can only express loops in which the repeated markings are explicitly stated, this is sufficient to encode several of the types of fairness given by Landweber [21] and Carstensen and Valk [8]. We now show that $\operatorname{MCP}\left(\tilde{\mathscr{L}}\left(Q^{\prime}, F, X\right)\right)$ for general PNs is equivalent to reachability. 


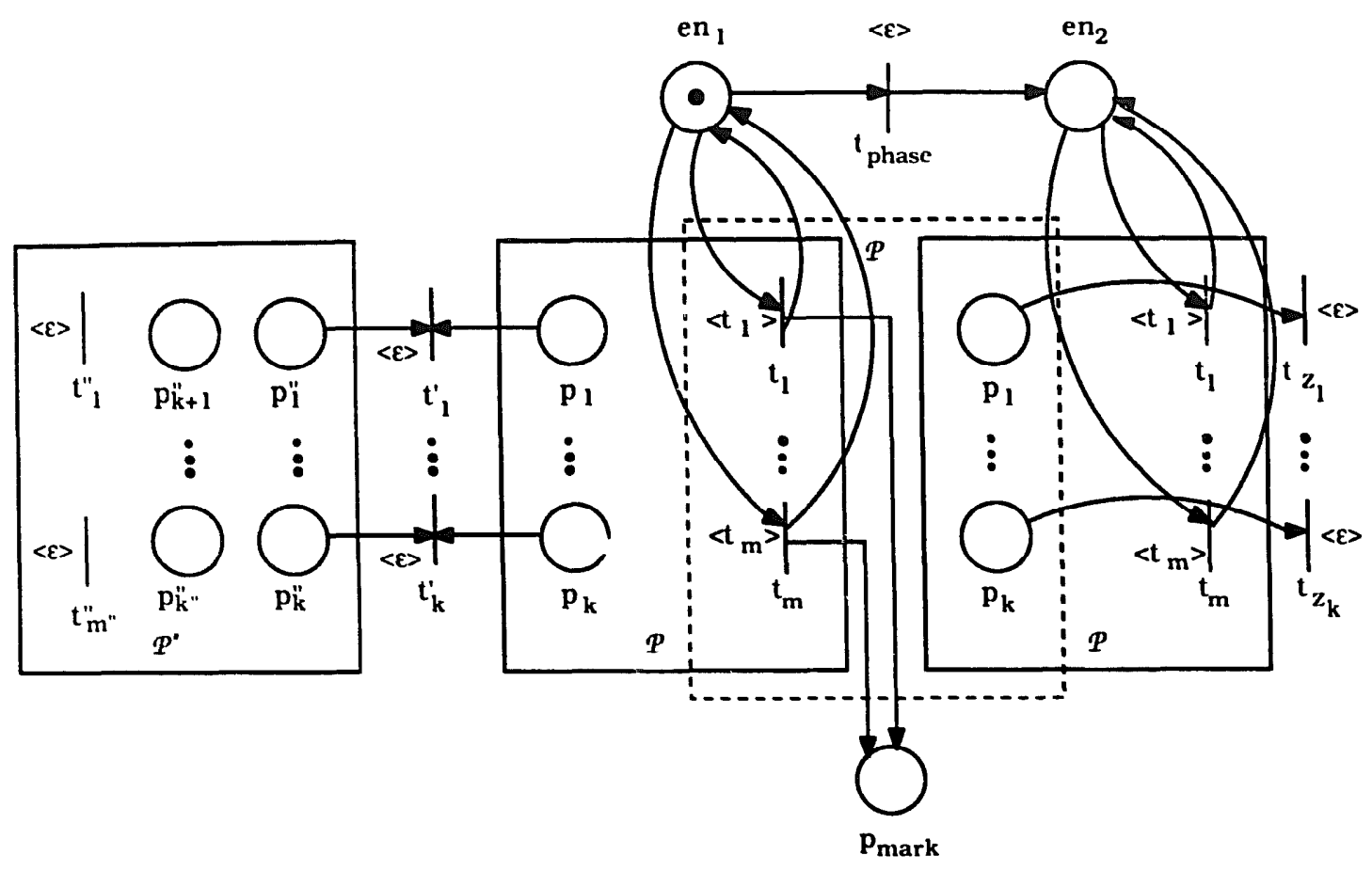

F. 3. $\mathscr{P}^{\prime}$ for $\neg \operatorname{co}(\mu)$.

Theorem 3.7. $\operatorname{MCP}\left(\tilde{\mathscr{L}}\left(Q^{\prime}, \boldsymbol{F}, \boldsymbol{X}\right)\right) \equiv{ }_{\text {PTIME }} \mathrm{RP}$.

Proof. We will first show $\mathrm{RP} \leqslant_{\mathrm{PTIME}} \operatorname{MCP}\left(\tilde{\mathscr{L}}\left(\boldsymbol{Q}^{\prime}, \boldsymbol{F}, \boldsymbol{X}\right)\right)$. Let $\mathscr{P}$ be an arbitrary PN and $\mu$ be an arbitrary marking of $\mathscr{P}$. We construct a PN $\mathscr{P}^{\prime}$ from $\mathscr{P}$ by adding a transition $t$ that does nothing and is always enabled. Clearly, $\mu \in R\left(\mathscr{P}^{\prime}\right)$ iff $\mu \in R(\mathscr{P})$, and any finite firing sequence in $\mathscr{P}^{\prime}$ can be made infinite. Since we can express reachability in $\tilde{\mathscr{L}}\left(Q^{\prime}, \boldsymbol{F}, \boldsymbol{X}\right)$ as in the proof of Theorem 3.6, $\mathbf{R P} \leqslant_{\text {PTIME }} \operatorname{MCP}\left(\tilde{\mathscr{L}}\left(Q^{\prime}, F, X\right)\right)$.

We will now show $\operatorname{MCP}\left(\tilde{\mathscr{L}}\left(Q^{\prime}, F, X\right)\right) \leqslant_{\text {PTIME }}$ RP. We will reduce thic MCP to $\operatorname{FMCP}\left(\tilde{\mathscr{L}}\left(Q^{\prime}, F, X\right)\right)$; the result will then follow from Theorem 3.6. Let $\mathscr{P}=$ $\left(P, T, \varphi, \mu_{0}\right)$ be an arbitrary $\mathrm{PN}$, and let $f$ be an arbitrary wff in $\tilde{\mathscr{L}}\left(Q^{\prime}, F, X\right)$. Let $f^{\prime} \equiv f \wedge F \wedge_{p \in P} \operatorname{lp}(p)$. It is a straightforward matter to show that $\mathscr{P}$ is a model for $f$ iff $\mathscr{P}$ is a finite model for $f^{\prime}$.

One question left open in [16] was whether $\operatorname{MCP}\left(\mathscr{L}^{x}\left(Q^{\prime}\right)\right)$ is decidable for conflict-free PNs. In Theorem 3.9, we will give a positive answer to this question by showing the problem with respect to general PNs to be equivalent to RP. Again, Theorem 3.9 may be viewed as a refinement of Theorem 3.6. Clearly, $\mathscr{L}^{\infty}\left(Q^{\prime}\right)$ can be used to express fairness constraints for which a certain event must be repeated infinitely often. We now define the following terminology. A set of wffs $\mathscr{F}$ is Q-pumpable iff there is a PTIME function $g: \mathscr{F} \rightarrow \tilde{\mathscr{L}}(Q, F, X)$ such that

(1) for any $f \in \mathscr{F}$, and any infinite firing sequence $\sigma$ in $\mathscr{P}$, if $\langle\mathscr{P}, \sigma, 0\rangle \vDash G F f$, then for any infinite set $I$ of natural numbers such that if $i \in I$, then $\langle\mathscr{P}, \sigma, i\rangle \vDash f$, there is an infinite subset $I^{\prime}$ of $I$ such that for any $i, j \in I^{\prime}, i<j,\langle\mathscr{P}, \sigma[j], i\rangle \vDash g(f)$; and 
(2) if $\mathscr{P}=\left(P, T, \varphi, \mu_{0}\right)$ is such that $\langle\mathscr{P}, \sigma, n\rangle \models g(f)$ for some finite $\sigma=\sigma[n] \sigma^{\prime}$, then $\mu_{0} \stackrel{\sigma[n]}{\longrightarrow} \mu_{1} \stackrel{a^{\prime}}{\rightarrow} \mu_{2} \stackrel{g}{\rightarrow} \cdots$ such that $\left\langle\left(P, T, \varphi, \mu_{j}\right), \sigma^{\prime}[1], 0\right\rangle \vDash f$ for all $j \geqslant 1$.

Before showing Theorem 3.9, we give the following lemma.

Lemma 3.8. The set of all positive Boolean combinations of predicates in $Q^{\prime} \cup \tilde{Q}^{\prime}$ is Q-pumpable.

Proof. Let $\mathscr{P}=\left(P, T, \varphi, \mu_{0}\right)$ be an arbitrary PN. We will show by induction on the structure of $f$ that we can construct in PTIME a $g(f)$ satisfying conditions (1) and (2) above.

Base Case 1: $f=\operatorname{ge}(p, c)$. Let $g(f)=\operatorname{ge}(p, c) \wedge \wedge_{p^{\prime} \in P} \operatorname{lp}\left(p^{\prime}\right)$. Let $\sigma$ be any infinite firing sequence such that $\langle\mathscr{P}, \sigma, 0\rangle \vDash G F f$, and let $I$ be any infinite set of natural numbers such that if $i \in I$, then $\langle\mathscr{P}, \sigma, i\rangle \models f$. Let $\mu_{i}$ be such thaî $\mu_{0} \stackrel{\text { ofi] }}{\longrightarrow} \mu_{i}$, and let $\theta$ be the sequence $\mu_{i_{1}}, \mu_{i_{2}}, \ldots$ such that $\left\{i_{1}, i_{2}, \ldots\right\}=I$ and $i_{1} \leqslant i_{2} \leqslant \cdots$. Since $\theta$ is infinite, there must be an infinite subsequence of $\theta, \theta^{\prime}=\mu_{j_{1}}, \mu_{j_{2}}, \ldots$ such that $\mu_{j_{1}} \leqslant \mu_{j_{2}} \leqslant \cdots$. Clearly, for any natural numbers $r$ and $s$ such that $r<s$, $\left\langle\mathscr{P}, \sigma\left[j_{s}\right], j_{r}\right\rangle \vDash g(f)$. Part (2) clearly holds.

Base Case 2: $f=\neg \operatorname{ge}(p, c) . g(f)=\neg \operatorname{ge}(p, c) \wedge z \mathrm{zl}(p) \wedge \wedge_{p^{\prime} \in P} \operatorname{lp}\left(p^{\prime}\right)$ clearly works.

Base Case 3: $f=\mathrm{fi}(t) . g(f)=\mathrm{f}(t) \wedge \bigwedge_{p^{\prime} \in P} \operatorname{lp}\left(p^{\prime}\right)$ clearly works.

Base Case 4: $f=\neg f(t) . g(f)=\neg f(t) \wedge \wedge_{p^{\prime} \in P} \backslash p\left(p^{\prime}\right)$ clearly works.

Now assume we have $f_{1}$ and $f_{2}$ for which conditions (1) ar. 1 (2) hold.

Case 5: $f=f_{1} \vee f_{2}$. Let $g(f)=g\left(f_{1}\right) \vee g\left(f_{2}\right)$. Let $\sigma$ be any infinite firing sequence such that $\langle\mathscr{P}, \sigma, 0\rangle \vDash G F f$, and let $I$ be any infinite set of natural numbers $i$ such that $\langle\mathscr{P}, \sigma, i\rangle \models f$. There must be some infinite subset $I^{\prime}$ of $I$ such that either $\forall i \in I^{\prime}$, $\langle\mathscr{P}, \sigma, i\rangle \models f_{1}$, or $\forall i \in I^{\prime},\langle\mathscr{P}, \sigma, i\rangle \vDash f_{2}$. Assume without loss of generality that $\forall i \in I^{\prime}$, $\langle\mathscr{P}, \sigma, i\rangle \models f_{1}$. From the induction hypothesis, there is an infinite subset $I^{\prime \prime}$ of $I^{\prime}$ such that for any $i, j \in I^{\prime \prime}, i<j,\langle\mathscr{P}, \sigma[j], i\rangle \models g\left(f_{1}\right)$. Then $\langle\mathscr{P}, \sigma[j], i\rangle \models g(f)$.

Now suppose $\mathscr{P}=\left(P, T, \varphi, \mu_{0}\right)$ such that $\langle\mathscr{P}, \sigma, n\rangle \models g(f)$ for some finite $\sigma=$ $\sigma[n] \sigma^{\prime}$. Then either $\langle\mathscr{P}, \sigma, n\rangle \models g\left(f_{1}\right)$ or $\langle\mathscr{P}, \sigma, n\rangle \models g\left(f_{2}\right)$. Without loss of generality, assume the former. From the induction hypothesis, $\mu_{0} \stackrel{\sigma[n]}{\longrightarrow} \mu_{1} \stackrel{g}{\rightarrow} \mu_{2} \stackrel{g^{\prime}}{\rightarrow} \ldots$ such that $\left\langle\left(P, T, \varphi, \mu_{j}\right), \sigma[1], 0\right\rangle \vDash f_{1}$ for all $j \geqslant 1$. Therefore, $\left\langle\left(P, T, \varphi, \mu_{j}\right), \sigma[1], 0\right\rangle \vDash f_{1} \vee$ $f_{2}=f$.

Case 6: $f=f_{1} \wedge f_{2}$. Let $g(f)=g\left(f_{1}\right) \wedge g\left(f_{2}\right)$. Let $\sigma$ be any infinite firing sequence such that $\langle\mathscr{P}, \sigma, 0) \vDash G F f$, and let $I$ be any infinite set of natural numbers $i$ such that $\langle\mathscr{P}, \sigma, i\rangle \models f$. Then for any $i \in I,\langle\mathscr{P}, \sigma, i\rangle \models f_{1}$. From the induction hypothesis, there is an infinite subset $I^{\prime}$ of $I$ such that for any $i, j \in I^{\prime}, i<j,\langle\mathscr{P}, \sigma[j], i\rangle \vDash g\left(f_{1}\right)$. Since $I^{\prime} \subseteq I$, for any $i \in I^{\prime},\langle\mathscr{P}, \sigma, i\rangle=f_{2}$. From the induction hypothesis, there is an infinite subset $I^{\prime \prime}$ of $I^{\prime}$ such that for any $i, j \in I, i<j,\langle\mathscr{P}, \sigma[j], i\rangle \models g\left(f_{2}\right)$. Consequently, $\langle\mathscr{P}, \sigma[j], i\rangle \vDash g(f)$.

Now suppose $\mathscr{P}=\left(P, T, \varphi, \mu_{0}\right)$ such that $\langle\mathscr{P}, \sigma, n\rangle \vDash g(f)$ for some finite $\sigma=$ $\sigma[n] \sigma^{\prime}$. Then $\langle\mathscr{P}, \sigma, n\rangle \models g\left(f_{1}\right)$ and $\langle\mathscr{P}, \sigma, n\rangle \vDash g\left(f_{2}\right)$. From the induction hypothesis, $\mu_{0} \stackrel{\sigma[n]}{\longrightarrow} \mu_{1} \stackrel{\sigma^{\prime}}{\rightarrow} \mu_{2} \stackrel{\sigma^{\prime}}{\rightarrow} \cdots$ such that $\left\langle\left(P, T, \varphi, \mu_{j}\right), \sigma^{\prime}[1], 0\right\rangle \models f_{1}$ and $\left\langle\left(P, T, \varphi, \mu_{j}\right)\right.$, $\left.\sigma^{\prime}[1], 0\right\rangle \models f_{2}$ for all $j \geqslant 1$. Therefore, $\left\langle\left(P, T, \varphi, \mu_{j}\right), \sigma^{\prime}[1], 0\right\rangle \vDash f_{1} \wedge f_{2}=f$. 
Theorem 3.9. $\operatorname{MCP}\left(\mathscr{L}^{\infty}\left(Q^{\prime}\right)\right) \equiv_{\text {PTIME }} R P$.

Proof. Let $G F f \in \mathscr{L}^{\infty}\left(Q^{\prime}\right)$. We can construct in PTIME an $f^{\prime} \equiv f$ such that negations occur only on predicates in $f^{\prime}$. Let $g$ be the PTIME function constructed in Lemma 3.8. It now follows immediately from the properties of $g$ that $\mathscr{P}$ is a model for $f$ iff $\mathscr{P}$ is a finite model for $g\left(f^{\prime}\right)$. Thus, MCP $(\mathscr{F}) \leqslant_{\text {PTIME }} R$ P. The construction given in Theorem 3.7 shows $R P \leqslant$ ptime $\operatorname{MCP}\left(\mathscr{L}^{\infty}\left(Q^{\prime}\right)\right)$.

\section{The fair nontermination problem}

In this section, we examine the complexities of $\mathbf{2 4}$ fair nontermination problems. We use the machinery developed in Section 3 to prove Theorems 4.2, 4.10, and 4.17, where a total of seven of these problems are shown to be equivalent to RP. Particularly in the latter two theorems, this machinery provides for very succinct reductions to RP, whereas "brute force" reductions are ruch longer and considerably more tedious. In the remainder of the theorems in this section, most of the problems we study are shown to be either equivalent to BP or $\Sigma_{1}^{1}$-complete. The results of this section are summarized in Section 5 (Table 1).

The first notions of fairness we consider were defined in [21] and [8]. These definitions of fairness are such that a fair firing sequence must visit certain predefined markings or transitions infinitely often. It is worth mentioning that the notion of "enabledness' does not play any role in these definitions (other than the fact that the definitions deal with firing sequences). Given an infinite firing sequence $\sigma=$ $t_{1} t_{2} \ldots$, we define inf ${ }^{\mathrm{M}}(\sigma)$ (inf $\left.^{\mathrm{T}}(\sigma)\right)$ to be the set of markings (transitions) that occur infinitely often in $\sigma$ (i.e., inf ${ }^{M}(\sigma)=\left\{\mu \mid\right.$ there are infinitely many $i$ such that $\left.\mu_{0} \stackrel{\sigma[i]}{\longrightarrow} \mu\right\}$ and $\inf ^{\mathrm{T}}(\sigma)=\left\{t_{i} \mid t_{i}\right.$ occurs infinitely often in $\left.\left.\sigma\right\}\right)$. Let $\mathscr{A}$ be a finite set of finite nonempty sets of markings. An infinite firing sequence $\sigma=t_{1} t_{2} \ldots$ is said to be

- M1-fair iff $\exists A \in \mathscr{A}, \exists i \in N: \mu_{0} \stackrel{\sigma[i]}{\longrightarrow} \mu \in A$ (i.e., some marking reached by $\sigma$ is in $A$ ).

- Ml'-fair iff $\exists A \in \mathscr{A}, \forall i \in N: \mu_{0} \stackrel{\sigma[i]}{\longrightarrow} \mu_{i} \in A$ (i.e., every marking reached by $\sigma$ is in $A$ ).

- M2-fair iff $\exists A \in \mathscr{A}$, inf ${ }^{\mathrm{M}}(\sigma) \cap A \neq \emptyset$ (i.e., some marking reached infinitely often by $\sigma$ is in $A$ ).

- $\mathrm{M} 2^{\prime}$-fair iff $\exists A \in \mathscr{A}$, $\inf ^{\mathrm{M}}(\sigma) \neq \emptyset$ and $\inf ^{\mathrm{M}}(\sigma) \subseteq A$ (i.e., $\sigma$ reaches some marking infinitely often and every marking reached infinitely often by $\sigma$ is in $A$ ).

- M3-fair iff $\exists A \in \mathscr{A}$, inf ${ }^{M}(\sigma)=A$ (i.e., the set of markings reached infinitely often by $\sigma$ is an elerent of $A$ ).

- M3'-fair iff $\exists A \in \mathscr{A}, A \subseteq \inf ^{\mathrm{M}}(\sigma)$ (i.e., every marking in $A$ is reached infinitely often by $\sigma$ ).

Similarly, let $\mathscr{A}$ be a finite set of nonempty subsets of transitions. $\sigma$ is said to be:

- T1-fair iff $\exists A \in \mathscr{A}, \exists i \in N^{+}: t_{i} \in A$.

- Tl'fair iff $\exists A \in \mathbb{A}, \forall i \in N^{+}: t_{i} \in A$. 
- T2-fuir iff $\exists A \in \mathscr{A}$, inf ${ }^{\mathrm{T}}(\sigma) \cap A \neq \emptyset$.

- T2 - rive iff $\exists A \in \mathscr{A}$, $\inf ^{\mathrm{T}}(\sigma) \subseteq A$.

- T3-fair iff $\exists A \in \mathscr{A}$, $\inf ^{\mathrm{T}}(\sigma)=A$.

- T3'-fair iff $\exists A \in \mathscr{A}, A \subseteq \inf ^{\mathrm{T}}(\sigma)$.

We will now investigate the complexity of the nontermination problems with respect to the above fairness constraints. The first theorem gives an exception to our general classification scheme due to the fact that for $M 1^{\prime}$-fairness the entire allowable reachability set is given as input to the problem.

\section{Theorem 4.1. $\mathrm{NTP}^{\mathrm{MI}}$ is NLOGSPACE-complete.}

Proof. The proof method ere is similar to the proof used in [16] to solve a similar problem for conflict-free Petri nets. For the sake of completeness, we sketch the proof in what follows. To show the problem to be in NLOGSPACE, we first guess an $\boldsymbol{A}$ in $\mathscr{A}$. Next, we check to make sure that the initial marking is in $A$. During the entire procedure, we will maintain a pointer to keep track of the current marking. (Note here that the amount of space needed to maintain such a pointer is logarithmic in the size of the input.) Initially, the pointer points to the initial marking. We then repeatedly guess a transition $t$ and a marking $\mu$ in $A$ and verify that $\mu$ can be produced by firing $t$ in the current marking. If so, $\mu$ becomes the current marking. If the above procedure can be performed for more than $|A|$ times, then there is an M1'-fair computation. Clearly, the above procedure is in NLOGSPACE. The problem was shown to be NLOGSPACE-hard for confiict-free PNs in [16]; thus, it must also be NLOGSPACE-hard for general PNs.

In showing the following theorem, we make use of the temperal logic results given in Theorems 3.7 and 3.9. This machinery makes the proof very succinct; however, this particular theorem is not exceedingly dificult to prove without using these results. The real power of our logic will be exploited in Theorems 4.10 and 4.17.

Theorem 4.2. $\mathrm{NTP}^{x} \equiv_{\mathrm{PTIME}} \mathrm{RP}$, for $x \in\left\{\mathrm{M} 1, \mathrm{M} 2, \mathrm{M} 2^{\prime}, \mathrm{M} 3, \mathrm{M}^{\prime}\right\}$.

Proof. We first show RP $\leqslant_{\text {ptime }}$ NTP ${ }^{x}$. We use a technique from [16]. Let $\mathscr{P}=$ $\left(P, T, \varphi, \mu_{0}\right)$ be an arbitrary $P N$ and $\mu$ be an arbitrary marking of $\mathscr{P}$. We now construct a Petri net $\mathscr{P}^{\prime}$ and a set $\mathscr{A}$ such that $\mu \in R(\mathscr{P})$ iff there is an $x$-fair computation with respect to $\mathscr{A}$ in $\mathscr{P}^{\prime}$. The new Petri net $\mathscr{P}^{\prime}$ is identical to $\mathscr{P}$ except that it has an additional transition that is always enabled and does nothing. Now let $\mathscr{A}=\{\{\mu\}\}$. Clearly, $\mu \in R(\mathscr{P})$ iff $\mathscr{P}^{\prime}$ has an $x$-fair computation with respect to $\mathscr{A}$, where $x \in\{\mathrm{M} 1, \mathrm{M} 2, \mathrm{M} 2$ ', M3, M3'\}. Furthermore, the reduction can be done in polynomial time.

We will now show NTP $^{x} \leqslant_{\text {PTIME }} R P$ for $x \in\left\{M 1, M 2, M 2^{\prime}, M 3, M 3^{\prime}\right\}$. Let $\mathscr{P}=$ $\left(P, T, \varphi, \mu_{0}\right)$ be an arbitrary $P N$, and let $\mathscr{A}$ be a finite set of nonempty finite sets of markings. For each element $A$ of $\mathscr{A}$, let $A=\left\{\mu_{A 1}, \mu_{A 2}, \ldots, \mu_{A k_{A}}\right\}$. We will give for 
each $x$ a wff $f_{x}$ such that $\mathscr{P}$ has an infinite $x$-fair path iff $\mathscr{P}$ is a mode: for $f_{x}$; furthermore, $f_{x}$ will be in either $\tilde{\mathscr{L}}\left(Q^{\prime}, F, X\right)$ or $\mathscr{L}^{\infty}\left(Q^{\prime}\right)$. The theorem will then follow from Theorems 3.7 and 3.9. We define:

- eq $(\mu) \equiv \bigwedge_{p \subset P}[\operatorname{ge}(p, \mu(p)) \wedge \neg \operatorname{ge}(p, \mu(p)+1)]$;

- $\operatorname{in}(A) \equiv \bigvee_{\mu \in A} e q(\mu)$;

- $f_{M 1} \equiv \bigvee_{A \in, \mathcal{A}}(\operatorname{in}(A) \vee F$ in $(A)$,

- $f_{\mathrm{M} 2} \equiv \bigvee_{A \in S} G F$ in $(A)$;

- $f_{\mathrm{M} 2} \equiv \bigvee_{A \in, A} F(\operatorname{in}(A) \wedge X(\operatorname{in}(A) \wedge X(\operatorname{in}(A) \ldots X(\operatorname{in}(A)) \ldots)))$ (there are $k_{A} X$ operators);

- $f_{\mathrm{M} 3^{3}} \equiv \bigvee_{A \epsilon, d} F\left(\operatorname{eq}\left(\mu_{A 1}\right) \wedge F\left(\operatorname{eq}\left(\mu_{A 2}\right) \wedge \cdots \wedge F\left(\operatorname{eq}\left(\mu_{A k_{A}}\right) \wedge F \operatorname{eq}\left(\mu_{A 1}\right)\right) \ldots\right)\right)$.

In order to define $f_{\mathrm{M} 3}$, we first construct for each $A \in \mathscr{A}$ a graph $G_{A}=\left(A, E_{A}\right)$, where $E_{A}=\{(u, v) \mid u, v \in A$ and for some $t \in T, u \rightarrow v\}$. We then let $\mathscr{A}^{\prime}=\left\{A \mid G_{A}\right.$ is strong!y connected $\}$. $\mathscr{A}^{\prime}$ can clearly be constructed in PTIME (see, e.g., [37]). We now define: - $f_{\mathrm{M} 3} \equiv V_{A \in, S^{\prime}} F$ eq $\left(\mu_{A 1}\right)$.

It should be clear that for each $x$, there is an infinite $x$-fair firing sequence in $\mathscr{P}$ iff $\mathscr{P}$ is a model for $\boldsymbol{f}_{\boldsymbol{x}}$.

We now turn our attention to the 6 transition-related types of fairness. The NTP', for $x \in\left\{\mathrm{T} 1, \mathrm{Tl}^{\prime}, \mathrm{T} 2, \mathrm{~T} 2^{\prime}, \mathrm{T} 3, \mathrm{~T} 3^{\prime}\right\}$, has been shown to be decidable in [38]. However, no complexity analysis was given there. In what follows, we show that these problems are equivalent to the BP. The proofs require showing the fact that if an infinite $x$-fair computation exists, then there must be a short "witness" to this fact. The proof of such a fact, generally speaking, is based on the method that Rackoff used in the complexity analysis of the BP in [31] (see also [32]).

A finite firing sequence $\sigma \sigma^{\prime}$ is self-covering iff $\mu_{0} \stackrel{a}{\rightarrow} \mu_{i} \stackrel{\sigma^{\prime}}{\rightarrow} \mu_{1}$ and $\mu_{j} \geqslant \mu_{i}$. (Note that $\sigma^{\prime}$ can be executed infinitely many times.) We define $T(\sigma)=\{t \mid$ transition $t$ occurs in $\sigma\}$. (Similarly, $T\left(\sigma^{\prime}\right)=\left\{t \mid\right.$ transition $t$ occurs in $\left.\sigma^{\prime}\right\}$.) Then we have the following easily shown lemma.

Lemma 4.3. Given a Petri net $\mathscr{P}$ and a finite set $\mathscr{A}$ of finite nonempty sets of transitions, there is an infinite $x$-fair firing sequence, where $x \in\left\{\mathrm{T1}_{1}, \mathrm{T1}^{\prime}, \mathrm{T} 2, \mathrm{~T} 2^{\prime}, \mathrm{T} 3, \mathrm{~T}^{\prime}\right\}$, iff there is a self-covering firing sequence $\sigma \sigma$ ' such that

(1) (T1-fair) $\exists A \in \mathscr{A}, A \cap T(\sigma) \neq \emptyset$,

(2) (T1'-fair) $\exists A \in \mathscr{A}, T(\sigma) \cup T\left(\sigma^{\prime}\right) \subseteq A$,

(3) (T2-fair) $\exists A \in \mathscr{A}, A \cap T\left(\sigma^{\prime}\right) \neq \emptyset$,

(4) (T2-fair) $\exists A \in \mathscr{A}, T\left(\sigma^{\prime}\right) \subseteq A$,

(5) (T3-fair) $\exists A \in \mathscr{A}, T\left(\sigma^{\prime}\right)=A$,

(6) (T3'-fair) $\exists A \in \mathscr{A}, A \subseteq T\left(\sigma^{\prime}\right)$.

We now give a lemma that allows us to derive an upper bound on the space complexity of $\mathrm{NTP}^{x}$, where $x \in\left\{\mathrm{T} 1, \mathrm{TI}^{\prime}, \mathrm{T} 2, \mathrm{~T}^{\prime}, \mathrm{T} 3, \mathrm{~T}^{\prime}\right\}$. The proof closely parallels the proof of Rackoff [31]; hence, it is omitted. 
Lemma 4.4. Given a transition $t$ and $a$ set of transitions $B$, the length of the shortes:

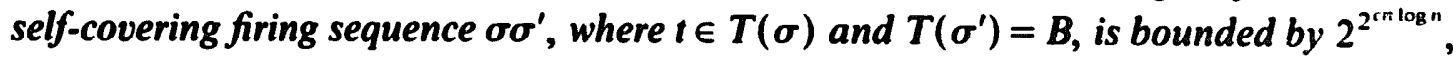
for some constant $c$ independent of $t, B$ and $n$.

In order to derive lower bounds for $\mathrm{NTP}^{x}, x \in\left\{\mathrm{T} 1, \mathrm{T1}^{\prime}, \mathrm{T} 2, \mathrm{~T}^{\prime}, \mathrm{T} 3, \mathrm{~T} 3^{\prime}\right\}$, we define the following problems, which are equivalent to $B P$ with respect to PTIME many-one reductions. (Note that these equivalences are not known to hold for LOG/LIN many-one reductions.)

- INF:

Instance: Given a Petri net $\mathscr{P}$,

Question: Is there an infinite firing sequence?

- CP:

Instance: Given a Petri net $\mathscr{P}$ and a marking $\mu$,

Question : Is there a $\mu^{\prime} \in R(\mathscr{P})$ such that $\mu \leqslant \mu^{\prime}$ ?

The following lemma follows from results of Rackoff [31] and Lipton [23].

Lemma 4.5. INF $\equiv_{\text {PTIME }} \mathrm{CP} \equiv_{\text {PTIME }} B P$.

We can now show the following.

Theorem 4.6. $\mathrm{NTP}^{x} \equiv_{\mathrm{PTIME}} \mathrm{BP}$, for $x \in\left\{\mathrm{T} 1, \mathrm{~T} 1^{\prime}, \mathrm{T} 2, \mathrm{~T}^{\prime}, \mathrm{T} 3, \mathrm{~T}^{\prime}\right\}$.

Proof. To show the NTP ${ }^{x} \leqslant_{\text {ptime }}$ BP, we note that by applying Lemmas 4.3 and 4.4, we have an NSPACE $\left(2^{\operatorname{cn} \log n}\right)$ upper bound for each problem. Since the BP is hard for NSPACE(2 ${ }^{\text {poly }}$ ) with respect to PTIME many-one reductions [23], NTP $^{x} \leqslant$ PTIME $B P$.

We now show BP $\leqslant_{\text {ptime }}$ NTP $^{x}$ for each $x$. Let $\mathscr{P}=\left(P, T, \varphi, \mu_{0}\right)$ be an arbitrary PN, and let $\mathscr{A}=\{T\}$. Clearly, $\mathscr{P}$ has an infinite $x$-fair firing sequence for $x \in$ $\left\{\mathrm{T} 1, \mathrm{T1}^{\prime}, \mathrm{T} 2, \mathrm{~T}^{\prime}, \mathrm{T}^{\prime}\right\}$ iff it has an infinite firing sequence. Thus, BP $\leqslant_{\text {PTIME }}$ INF $\leqslant_{\text {PTIME }}$ NTP $^{x}$ for $x \in\left\{T 1, T 1^{\prime}, T 2, T^{\prime}, T^{\prime}\right\}$.

To show BP $\leqslant_{\text {PTIME }}$ NTP $^{\text {T3 }}$, we will use the fact that $C P=$ prime $B P$. Let $\mathscr{P}$ be an arbitrary $\mathrm{PN}$, and let $\mu$ be an arbitrary marking. We construct $\mathscr{P}^{\prime}$ by adding to $\mathscr{P}$ a new transition $t$ which does nothing but which is only enabled at markings $\mu^{\prime} \geqslant \mu$. Let $\mathscr{A}=\{\{t\}\}$. Clearly, $\mathscr{P}^{\prime}$ has an infinite T3-fair firing sequence iff there is a $\mu^{\prime} \in R(\mathscr{P})$ such that $\mu \leqslant \mu^{\prime}$. Thus, BP $\leqslant_{\text {PTIME }} C P \leqslant_{\text {PTIME }} N_{T P}{ }^{T 3}$.

We now examine the NTP with respect to several notions of fairness in which the constraints are imposed in an implicit fashion, instead of by an explicit listing of the markings and/or transitions that a "fair" firing sequence must visit. We will first examine three types introduced in [22] and two extensions given in [7] (see also [8]). Given a Petri net $\mathscr{P}$ and a set of subsets of transitions $\mathscr{T}$, an infinite firing sequence $\sigma$ is said to be:

- impartial iff every transition in $\mathscr{P}$ occurs infinitely often in $\sigma$;

- just iff every transition that is enabled almost everywhere in $\sigma$ occurs infinitely often in $\sigma$; 
- fair iff every transition that is enabled infinitely often in $\sigma$ occurs infinitely often in $\sigma$;

- $f d p$ with respect to $\mathscr{T}$ (fdp- $\mathscr{T}$ ) iff for every $T \in \mathscr{T}$, if almost everywhere in $\sigma$ some $t$ in $T$ is enabled, then some $t^{\prime}$ in $T$ occurs infinitely often in $\sigma$ (here, fdp stands for finite delay property);

- fair with respect to $\mathscr{T}$ (fair- $\mathscr{T}$ ) iff for every $T \in \mathscr{T}$, if some $t$ in $T$ is enabled infinitely often in $\sigma$, then some $t^{\prime}$ in $T$ occurs infinitely often in $\sigma$.

Theorem 4.7. $\mathrm{NTP}^{\mathrm{imp}} \equiv_{\text {PTIME }}$ BP.

Proof. It is not hard to see that NTP'mp is a special case of $\mathrm{NTP}^{\mathrm{T} 3}$ (by letting $\mathscr{A}$ contain only the set of all transitions); hence, from Theorem 4.6, $\mathrm{NTP}^{\text {imp }} \leqslant_{\text {PTIME }} \mathrm{NTP}^{\mathrm{T3}} \leqslant_{\text {PTIME }}$ BP. In order to show that BP $\leqslant_{\text {PTIME }} \mathrm{NTP}^{\mathrm{imp}}$, we will use the fact that $\mathrm{CP} \equiv_{\mathrm{PTIME}} \mathrm{BP}$. Let $\mathscr{P}=\left(P, T, \varphi, \mu_{0}\right)$ be an arbitrary PN, and let $\mu$ be an arbitrary marking. We construct $\mathscr{P}^{\prime}=\left(P, T^{\prime}, \varphi^{\prime}, \mu_{0}\right)$ by adding to $\mathscr{P}$ a new transition $t^{\prime}$ such that:

- $\varphi\left(p, t^{\prime}\right)=\mu(p)$ for all $p \in P$; and

- $\varphi\left(t^{\prime}, p\right)=\mu(p)+\sum_{t \in T} \varphi(p, t)$ for all $p \in P$.

Clearly, there is ar: infinite impartial firing sequence in $\mathscr{P}^{\prime}$ iff there is a $\mu^{\prime} \in R(\mathscr{P})$ such that $\mu^{\prime} \geqslant \mu$.

In order to show the next theorem, we define the single-place zero-reachability problem, which is equivalent to RP.

- RP-SO:

Instance: A PN $\mathscr{P}$ with a designated place $p$,

Question: Is there a $\mu \in R(\mathscr{P})$ such that $\mu(p)=0$ ?

The following lemma is well known; see, e.g., [28].

Lemma 4.8. RP-SO $\equiv_{\text {PTIME }}$ RP.

At this time, we are unable to show either NTP ${ }^{\text {just }}$ or NTP ${ }^{\text {rdp. }-9}$ to be decidable.' The main difficulty, we feel, is due to the fact that these fairness properties are nonmonotonic in the sense that the existence of a just (fdp- $\mathscr{T}$, respectively) firing sequence starting in $\mu$ by no means guarantees a just (fdp- $\mathscr{T}$ ) firing sequence starting in any $\mu^{\prime}$, where $\mu^{\prime} \geqslant \mu$. At the same time, we are unable to enforce zero-testing using either of these properties. However, we are able to show the following three theorems.

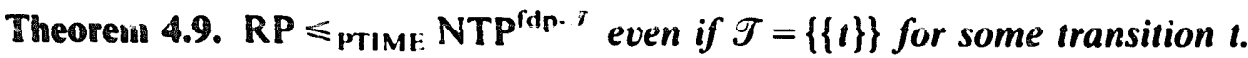

Proof. We will use the fact that RP-S0 $\equiv_{\text {PTIME }}$ RP. Let $\mathscr{P}=\left(P, T, \varphi, \mu_{0}\right)$ be a PN with a designated place $p_{1}$. We will construct $\mathscr{P}^{\prime}$ as shown in 'rig. 4. There is clearly

\footnotetext{
' Recently, Jančar [19] has claimed NTP ${ }^{\text {(dp. } . T}$ (and hence NTP ${ }^{u s t}$ ) to be decidable. The proof is an exponential-ume reduction to RP; thus, it comes very close to the lower bound that we show.
} 


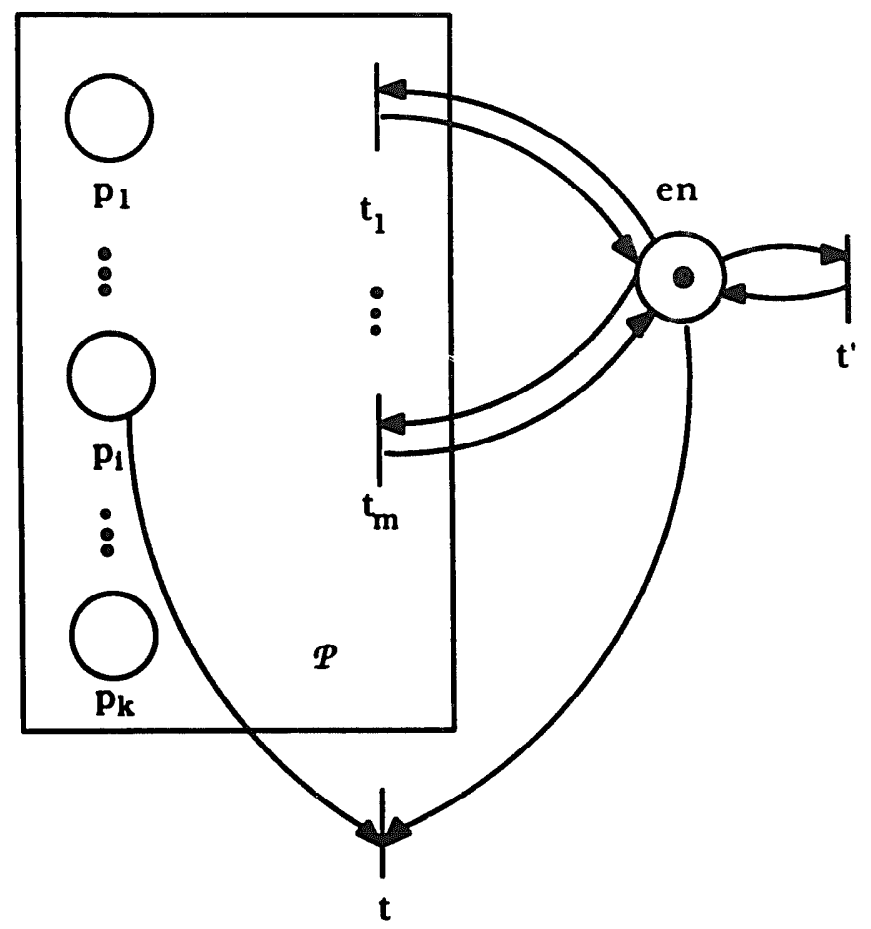

Fig. 4. $\mathscr{P}^{\prime}$ for fdp- $\mathscr{T}, \mathscr{T}=\{\{t\}\}$.

no infinite firing sequence in $\mathscr{P}^{\prime}$ in which $t$ fires. Thus, in any infinite firing sequence that is fdp with respect to $\{\{t\}\}, t$ must be disabled infinitely often; i.e., a marking $\mu$ must be reached such that $\mu\left(p_{i}\right)=0$. On the other hand, if $\mu_{0} \stackrel{g}{\rightarrow} \mu, \mu\left(p_{i}\right)=0$, then $\sigma t^{\prime} t^{\prime} t^{\prime} \ldots$ is fdp with respect to $\{\{t\}\}$. Therefore, RP $\leqslant$ PTIME RP-SO $\leqslant$ PTIME NTP ${ }^{\text {fdp- } \cdot \text {. }}$.

In [7], Carstensen showed that $\mathrm{NTP}^{\text {fdp } \cdot \bar{J}}$ is decidable if $|\mathscr{T}|=1$. We are able to improve upon this result by showing the problem to be equivalent to RP. In this proof, as opposed to Theorem 4.2 , the real power of the machinery developed in Section 3 is exploited. Without the umbrella of Theorem 3.6 and its subsequent refinement in Theorem 3.9, this proof would have been much longer and considerably more tedious. As it is, we are able to give a very succinct proof of an unobvious theorem.

Theorem 4.10. NTP fdp.. $\mathcal{J}^{\text {PTIME }}$ RP if $|\mathscr{T}|=1$.

Proof. From Theorem 4.9, we need only show that NTP ${ }^{\text {fdp. }} \leqslant_{\text {mime }}$ RP if $|\mathscr{T}|=1$. Let $\mathscr{P}=\left(P, T, \varphi, \mu_{0}\right)$ be an arbitrary $P N, T^{\prime} \subseteq T$, and $\mathscr{T}=\left\{T^{\prime}\right\}$. An infinite firing sequence $\sigma$ is fdp with respect to $\mathscr{T}$ iff

$$
\left.\langle\mathscr{P}, \sigma, 0\rangle \vDash G \mathbb{F}\left[\left(\bigwedge_{t \in T} \neg\left(\bigwedge_{p \in P} \operatorname{ge}(p, \varphi(p, t))\right)\right) \vee\left(\bigvee_{t \in T} f(t)\right)\right)\right] .
$$

Thus, from Theorem 3.9, NTP ${ }^{\text {fdp } . \mathcal{F}} \leqslant$ pTIME $\operatorname{RP}^{\mathrm{P}}$ if $|\mathcal{T}|=1$. 
The following theorem gives a lower bound for NTP ${ }^{\text {just }}$.

Theorem 4.11. RP $\leqslant_{\text {PTIME }}$ NTP ${ }^{\text {just }}$.

Proof. We will use the fact that $R P \leqslant_{\text {ptime }} N P^{f d p-\{1+\}\}}$. Let $\mathscr{P}=\left(P, T, \varphi, \mu_{0}\right)$ be an arbitrary PN, and let $t_{i}$ be an arbitrary iransition in $T$. We construct $\mathscr{P}^{\prime}$ as shown in Fig. 5. Suppose $\sigma$ is an infinite path in $\mathscr{P}$ that is fdp with respect to $\left\{\left\{t_{i}\right\}\right\}$. We construct $\sigma^{\prime}$ by inserting infinitely often in $\sigma t_{j}^{\prime} t_{j}^{\prime \prime}$ for all $j \neq i, 1 \leqslant j \leqslant m$; since en is incremented infinitely often, this can clearly be done. Clearly, $\sigma^{\prime}$ is an infinite firing sequence in $\mathscr{P}^{\prime}$ that is fdp with respect to $\left\{\left\{t_{i}\right\}\right\}$. Furthermore, for all $j \neq i, 1 \leqslant j \leqslant m$, $t_{j}^{\prime}$ and $t_{j}^{\prime \prime}$ fire infinitely often, and $t_{j}$ is disabled infinitely often. Therefore, $\sigma^{\prime}$ is just.

Now suppose $\sigma$ is an infinite just firing sequence in $\mathscr{P}^{\prime}$. In order for $\sigma$ to be infinite, there clearly must be infinitely many occurrences of transitions from $T$. Therefore, by deleting all occurrences of transitions not in $T$, we construct an infinite firing sequence $\sigma^{\prime}$ that is fdp with respect to $\left\{\left\{t_{i}\right\}\right\}$. Therefore, $R P \leqslant_{\text {PTIME }} N T P^{f d p-\{\{r\}\}} \leqslant_{\text {PTIME }} N^{\text {NTP }}{ }^{\text {just }}$.

In [7], Carstensen showed the NTP ${ }^{\text {fair }}$ and the NTP ${ }^{\text {fair- }-\overline{5}}$ to be undecidable. In what follows, we improve this result by showing both to be complete for $\Sigma_{1}^{1}$-the first level of the analytical hierarchy. We will later use these results to show a third

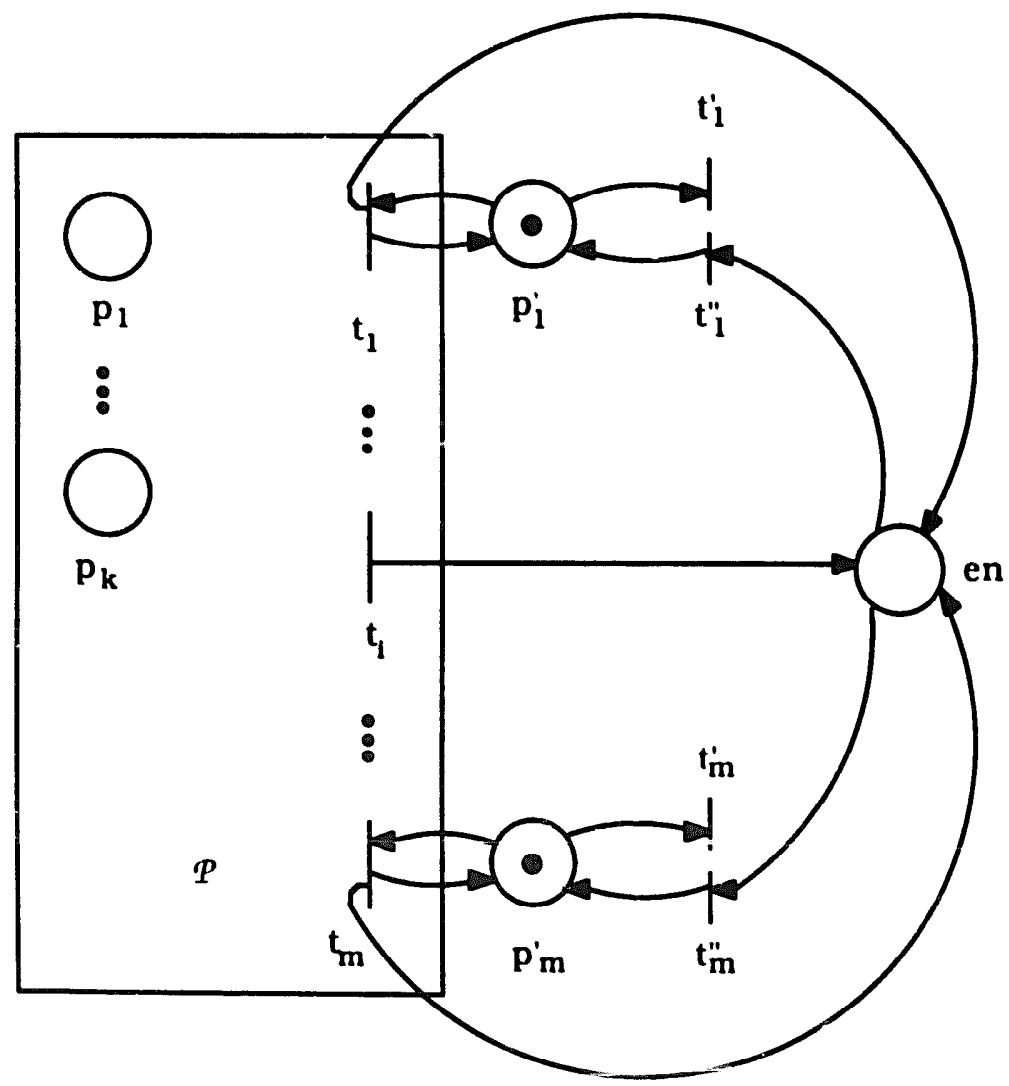

Fig. 5. $\boldsymbol{P}^{\prime}$ for justice. 
version to be $\Sigma_{1}^{\prime}$-complete. Our proofs involve certain generalized counter machines. We define an infinite-branching counter machine (ICM) to be a counter machine with the added capability to nondeterministically add to a counter any natural number in one step. The following lemma follows from [9].

Lemma 4.12. The set of all ICMs that contain an infinite computation on $\varepsilon$ is $\Sigma_{1}^{1}$-complete.

We are now ready to show that NTP fair and NTP fair. are $\Sigma_{1}^{1}$-complete.

Theorem 4.13. $\mathrm{NTP}^{\text {fair- } \mathcal{T}}$ is $\Sigma_{1}^{1}$-hard even if $\mathscr{T}$ contains only singleton sets.

Proof. Let $M$ be an arbitrary ICM. We will construct a $P N \mathscr{P}=\left(P, \Gamma, \varphi, \mu_{0}\right)$ and a set $\mathscr{T}$ of singleton subsets of $T$ such that $\mathscr{P}$ has an infinite firing sequence that is fair with respect to $\mathscr{T}$ iff $M$ has an infinite computation on $\varepsilon$. $\mathscr{P}$ must be able to simulate infinite-branching and zero-testing. The way we construct $\mathscr{P}$, zero-testing will be guaranteed to be coirectly simulated only after some finite (but unknown) amount of time. Hence, we will first construct an ICM $M^{\prime}$ to simulate $M$ such that for any configuration $I$ of $M^{\prime}$ (whether reachable or not), any infinite computation of $M^{\prime}$ from $I$ simulates an infinite computation of $M$. We will then construct $\mathscr{P}$ to simulate $M^{\prime}$.

We construct $M^{\prime}$ to repeatedly simulate finite subcomputations of $M$ as follows. $M^{\prime}$ will have one set of counters to simulate $M$, one set of counters to store the final counter values from the previous simulation of $M$, two counters to simulate a clock (one for time remaining, the other for the initial value), and a fixed number of scratch counters. The finite-state control of $M^{\prime}$ will store the state of $M$ in the current simulation and the final state from the previous simulation. Initially, the previous final configuration is the initial configuration of $M$, and the clock value is zero. $M^{\prime}$ iterates the following loop:

(1) Let $n$ be the value of the clock.

(2) Simulate $M$ for $n$ steps; halt if $M$ does.

(3) If the final configuration of $M$ differs from the previous final configuration, then halt.

(4) Simulate $M$ for one more step.

(5) Save configuration of $\boldsymbol{M}$.

(6) Reset clock to $n+1$.

(7) Restore $M$ to initial configuration.

Suppose $M^{\prime}$ starts in some arbitrary configuration $I$, and suppose there is an infinite computation $\sigma$ from $I$. Clearly, each ol the seven steps above must terminate, so each step occurs infinitely often in $\sigma$. Eventually, $\sigma$ must reach Step 7 . After this, when it reaches Step 2, it simulates a computation $\sigma^{\prime}$ of $M$ for $n$ steps, where $n$ is some natural number. Since $\sigma$ is infinite, it will not halt in Step 3. Each subsequent execution of Step 4 extends $\sigma^{\prime}$ by one move; hence, $\sigma$ simulates an infinite computation $\sigma^{\prime}$ of $M$. 
We now construct $\mathscr{P}$ to simulate $M^{\prime}$ using places to simulate states and counters in the obvious way, and transitions to simulate all moves except those of the form " $q_{1}$ : if $c_{i}=0$ then go to $q_{2}$ " and " $q_{1}$ : add some natural number to $c_{i}$ and go to $q_{2}$." Carstensen [7] has shown how these moves can be implemented; the constructions are shown in Figs. 6 and 7, respectively. Regarding Fig. 6, since $\left\{t_{2}\right\} \in \mathscr{T}, t_{1}$ can be fired while $c_{i}>0$ only finitely many times in any firing sequence that is fair with respect to $\mathscr{T}$. Likewise, for Fig. 7 , if $t_{2}$ is enabled, it must eventually fire in any

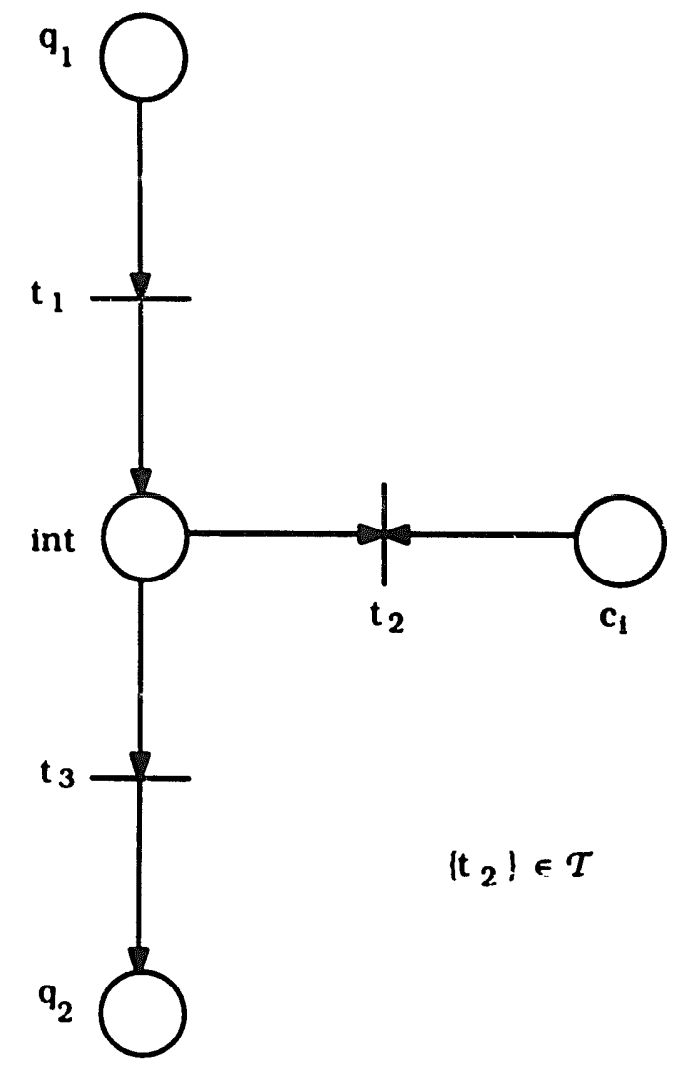

Fig. 6. Implementation of zero-testing for fair- $T$.

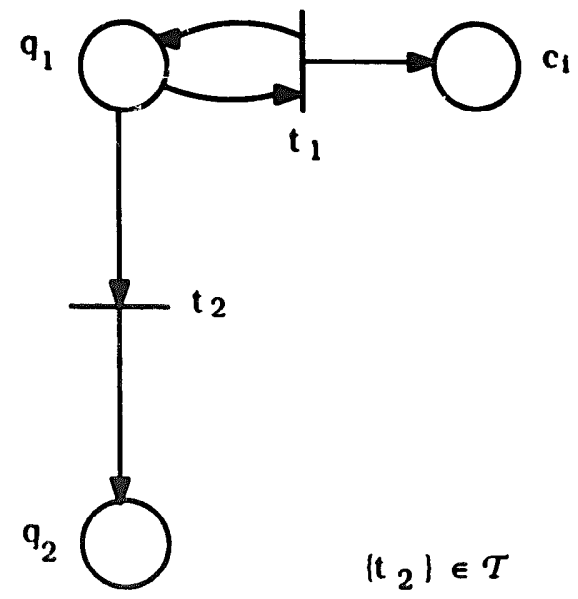

Fig. 7. Implementation of infinite-branching for fair- $J$. 
infinite firing sequence that is fair with respect to $\mathscr{T}$. Clearly, if $M$ has an infinite computation on $\varepsilon$, then $\mathscr{P}$ has an infinite firing sequence that is fair with respect to $\mathscr{T}$. Suppose, on the other hand, that $\mathscr{P}$ has an infinite firing sequence $\sigma$ that is fair for $\mathscr{T}$. After some point in $\sigma, \sigma$ correctly simulates $M^{\prime}$ from some initial configuration I. Since this simulation is infinite, $M$ must have an infinite computation.

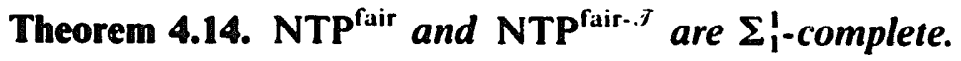

Proof. NTP ${ }^{\text {fair. } . j}$ was shown to be $\Sigma_{i}$-hard in Theorem 4.13. Carstensen [7] gives a

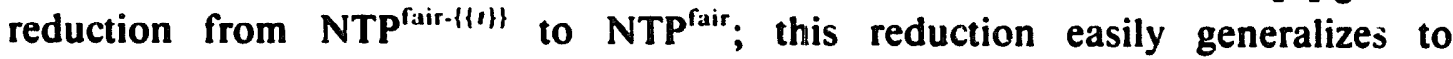
NTP $^{\text {fair- } \mathcal{T}} \leqslant$ PTIME NTP fair if $\mathscr{T}$ contains only singleton sets. Thus, from Theorem 4.13, NTP fair is also $\Sigma_{1}$-hard. See the proof of Theorem 4.15 below for an even more general version of this reduction.

Since NTP ${ }^{\text {fair }}$ is a restriction of NTP fair. 3 , we only need to show that NTP ${ }^{\text {fair. } . J} \in \Sigma_{1}$. Let $\mathscr{P}=\left(P, T, \varphi, \mu_{0}\right)$ be an arbitrary $P N$, and let $\mathscr{T}$ be a set of subsets of $T$. Clearly an infinite firing sequence $\sigma$ is fair with respect to $\mathscr{T}$ iff it can be divided into a sequence of finite firing sequences $\sigma_{0} \sigma_{1} \ldots$ such that for some subset $\mathscr{T}^{\prime}$ of $\mathscr{T}$, every set in $\mathscr{T}^{\prime}$ has at least one element in each $\sigma_{i}, i \geqslant 1$, and no transition in any set in $\mathscr{T} \backslash \mathscr{T}^{\prime}$ is enabled at any time in $\sigma$ after $\sigma_{0}$. We therefore construct an ICM $M$ to nondeterministically generate $\sigma$ as follows. $M$ first guesses $\mathscr{T}^{\prime}$. It thu guesses (in one step) $n_{0}$, the length of $\sigma_{0}$. Next, $M$ nondeterministically generates a firing sequence $\sigma_{0}$ of length $n_{0}$. For $i=1,2, \ldots, M$ :hen guesses $n_{i}$, the length of $\sigma_{i}$, and generates $\sigma_{i}$, verifying that it satis es the conditions outlinsd above. Clearly, $M$ has an infinite computation iff $\mathscr{P}$ has an infinite firing sequence that is fair with respect to $\mathscr{T}$. Therefore, NTP ${ }^{\text {fair }}$ and NTP ${ }^{\text {fair. } . \mathcal{J}}$ are $\Sigma_{\text {i-complete. }}^{\prime}$

In [7], Carstensen a!so considered fairness for bounded PNs. Given a PN $\mathscr{P}$, we say an infinite firing sequence $\sigma$ is

- bd-fair if $\mathscr{P}$ is bounded and $\sigma$ is fair.

Although NTP ${ }^{\text {bd-fair }}$ is clearly decidable and as hard as BP, no tighter bounds have been given for the problem. We now show the problem to be nonprimitive recursive.

Theorem 4.15. NTP ${ }^{\text {hd.fair }}$ is not primitive recursive.

Proof. We first define the following functions:

$$
\begin{aligned}
& f_{1}(n)=2 n, \\
& f_{1}(n)=f_{i=1}^{(n)}(1) \quad \text { for } i>1, \\
& f(n)=f_{n}(n),
\end{aligned}
$$

where $f_{1-1}^{(n)}$ denotes the $n$ th-fold composition of $f_{1-1}$. It can be shown that $f$ is not primitive recursive. Let $M$ be an arbitrary $f(n)$ time bounded TM, and let $x$ be an arbitrary input for $M$. Clearly, wa can construct in PTiME a 2-counter machine $M^{\prime}$ 
that simulates $M$ in $f(c n)$ time for some constant $c$. Carstensen [7] gave a PTIME construction of an unbounded PN $\mathscr{P}$ with a designated transition $t$ such that $\mathscr{P}$ had an infinite firing sequence that was fair with respect to $t$ iff $M^{\prime}$ halted on $x$. (In his construction, $\mathscr{P}$ simulated an arbitrary 2 -counter machine.) $\mathscr{P}$ operated by first guessing $m$, the number of steps executed by $M$, then repeatedly simulating $M$ on $\boldsymbol{x}$. If $\boldsymbol{M}$ did not halt in $\boldsymbol{m}$ steps, $\mathscr{P}$ would halt. $\mathscr{P}$ contained three potentially unbounded places: the one containing $\boldsymbol{m}$ and two containing counter values. $C$ !early, since $M^{\prime}$ is $f(c n)$ time bounded, these places need not exceed $f(c n)$ to simulate $M^{\prime}$. In [17], we gave a PTIME construction of a bounded PN which could produce a value of $f(c n)$ in a designated place (see also [26]). We can clearly use this construction to bound the three places in question by some number at least $f(c n)$. The details are left to the reader.

We will now show that given a bounded PN $\mathscr{P}$ and a set $\mathscr{T}$ of singleton subsets of transitions, we can construct in PTIME a bounded PN $\mathscr{P}^{\prime}$ that has an infinite bd-fair firing sequence iff $\mathscr{P}$ has an infinite firing sequence that is fair with respect to $\mathscr{T}$. This construction is a generalization of one given in [7], and is shown in Fig. 8. Note that the added places, $p_{1}^{\prime}, \ldots, p_{m}^{\prime}, p_{1}^{\prime \prime}, p_{2}^{\prime \prime}$, must always contain a total of three tokens; hence $\mathscr{P}^{\prime}$ remains bounded. Suppose $\mathscr{P}$ has an infinite path $\sigma$ that is fair with respect to $\mathscr{T}$. Prior to each occurrence of $t_{i}$ in $\sigma, 1 \leqslant i \leqslant j$, we can insert

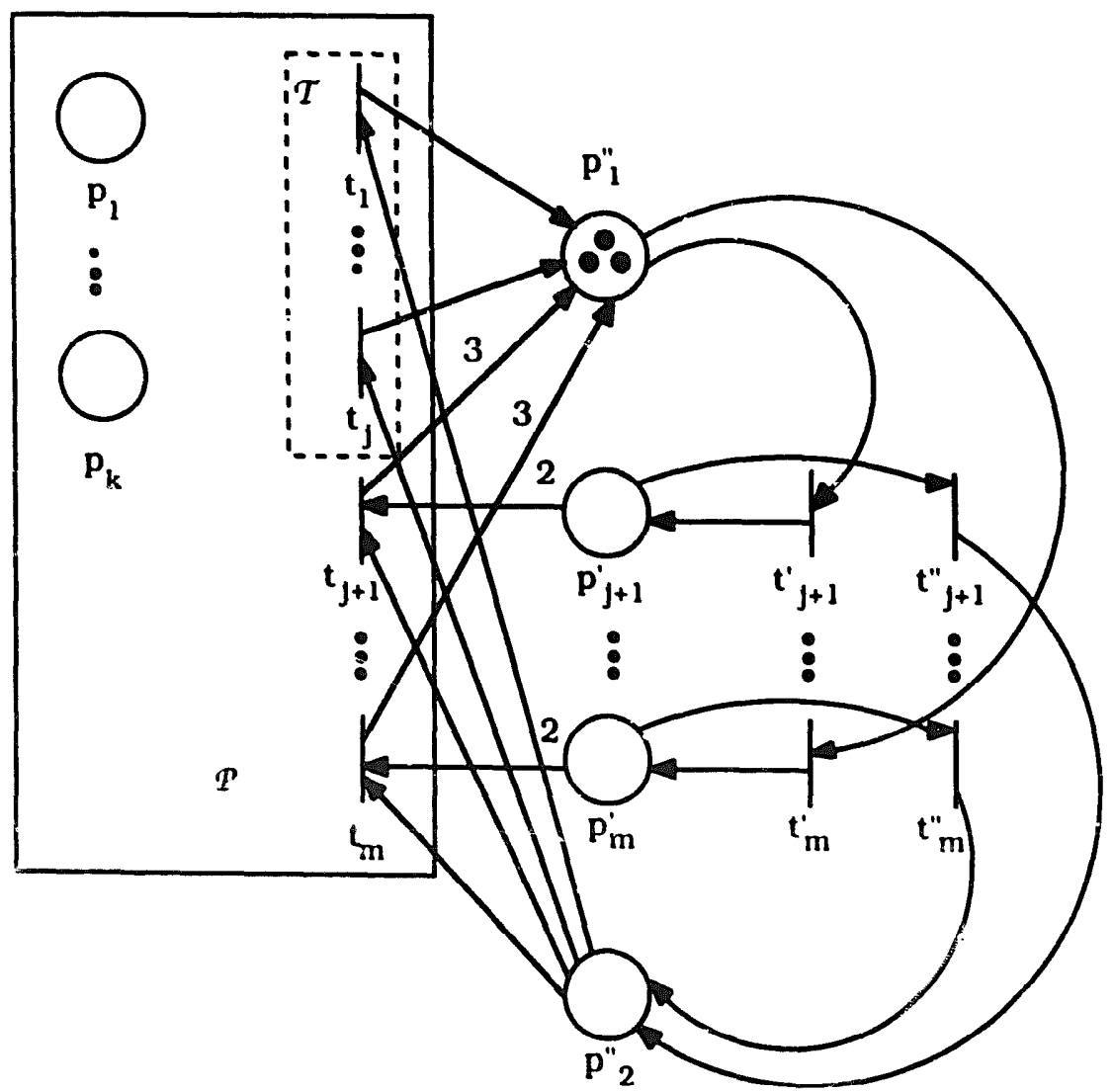

Fig. 8. $\Phi^{\prime}$ for bd-fairness. 
the sequence $t_{i}^{\prime} t_{i}^{\prime \prime}$ for some $i^{\prime}$, and prior to each occurrence of $t_{i}, j+1 \leqslant i \leqslant m$, we can insert the sequence $t_{i}^{\prime} t_{i}^{\prime} t_{i}^{\prime} \cdot t_{i}^{\prime \prime}$ for some $i^{\prime}$. It is easy to see that this new firing sequence is bd-fair. On the other hand, s ppose there is an infinite firing sequence $\sigma$ in $\mathscr{P}^{\prime}$ that is bd-fair. $\mathrm{Si}_{1}$ se $\boldsymbol{p}_{2}^{\prime \prime}$ must be positive before any transition in $\mathscr{P}$ can fire, by removing all occurrences of transitions not in $\mathscr{P}$, we clearly get an infinite firing sequence that is fair with respect to $\mathscr{T}$.

In [5], Best extended the definition of fairness using the notions of $i(\infty)$-enabledness. A transition $t$ is said to be $i$-enabled (or $\infty$-enabled if $i=\infty$ ) at a marking $\mu$ if there is a firing sequence $\sigma$ no longer than $i$ transitions such that $\mu \stackrel{\sigma}{\rightarrow} \mu^{\prime}$, and $t$ is enabled at $\mu^{\prime}$. For $1 \leqslant i \leqslant \infty$, an infinite computation $\sigma$ is said to be:

- $i$-fair iff for every transition $t$, if $t$ is $i$-enabled in infinitely many markings in $\sigma$, then $t$ occurs infinitely often.

(Note that "0-fairness" coincides with "fairness" as defined in [22]. Also, an equivalent definition for $\infty$-fairness was given in [30].)

In what follows, we show that NTP $^{i \text { fair }}, 0 \leqslant i<\infty$, is $\Sigma_{1}^{\prime}$-complete, but that NTP $^{x \text {-fair }}$ is equivalent to RP.

Theorem 4.16. For every $i, 0 \leqslant i<\infty$, NTP ${ }^{i-f a i r}$ is $\Sigma_{i}^{1}$-complete.

Proof. To show the lower bound, we will use a reduction from NTP fair. Let $\mathscr{P}$ be an arbitrary PN. We construct $\mathscr{P}^{\prime}$ as shown in Fig. 9. Clearly, $\mathscr{P}$ has an infinite fair firing sequence iff $\mathcal{P}^{\prime}$ has an infinite $i$-fair firing sequence. To show the upper bound we can clearly employ a similar strategy to that of the upper bound in Theorem 4.14, since $i$-enabledness is clearly a decidable property.

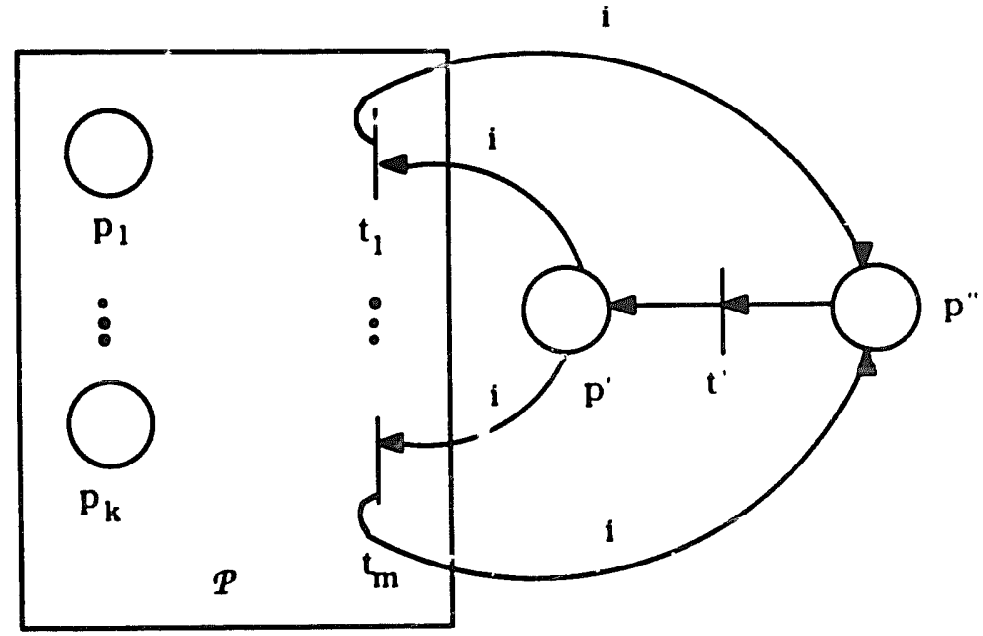

Fig. 9. $p^{\prime}$ for i-fair. 
The following theorem is the last in which we use the machinery developed in Section 3. In this theorem, we use Theorem 3.6 directly to give a succinct reduction to RP. As in Theorem 4.10, a direct reduction to RP would have been much more tedious.

Theorem 4.17. NTP ${ }^{\alpha-\text {-fair }} \equiv_{\text {PTIME }}$ RP.

Proof. We first show NTP ${ }^{\alpha-\text {-rair }} \leqslant_{\text {PrIme }}$ RP. Let $\mathscr{P}=\left(P, T, \varphi, \mu_{0}\right)$ te an arbitrary PN.

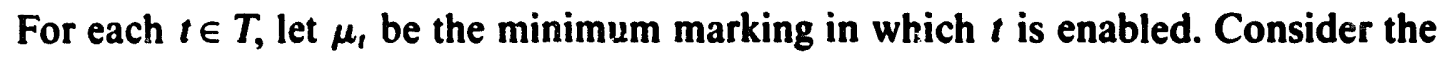
wff

$$
f=F\left[\bigwedge_{\in \in T}\left(\neg \operatorname{co}\left(\mu_{t}\right) \vee F f(t)\right) \wedge \bigwedge_{p \in P} \operatorname{lp}(p)\right]
$$

Clearly there is an infinite $\infty$-fair firing sequence in $\mathscr{P}$ iff $\mathscr{P}$ is a finite model for $f$. Thus, from Theorem 3.6, NTP ${ }^{\text {x-fair }} \leqslant$ PTIME RP.

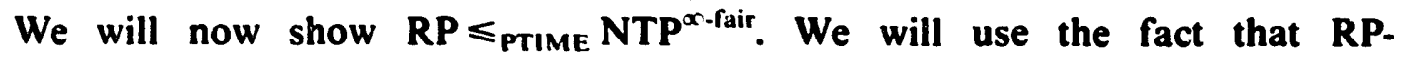
S0 $\equiv_{\text {ptIME }} R P$. Let $\mathscr{P}=\left(P, T, \varphi, \mu_{0}\right)$ be an arbitrary PN with a designated place $p_{i}$. We construct $\mathscr{P}^{\prime}$ as shown in Fig. 10. Suppose $\mathscr{P}^{\prime}$ has an infinite $\infty$-fair firing sequence. Since $t_{1}^{\prime}$ is enabled until it fires, it must eventually fire. Clearly, $t_{3}^{\prime}$ cannot

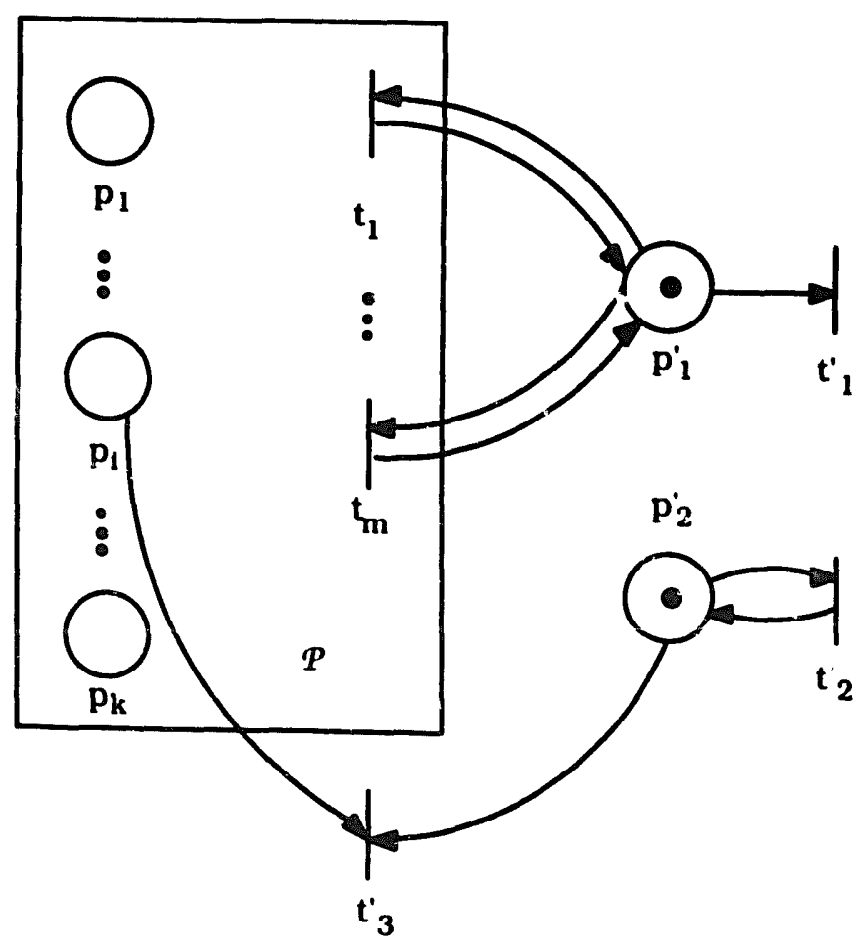

Fig. 10. $\rho^{\prime}$ for $x$-fair, state-fair ard pred-fair. 
fire in any infinire $\infty$-fair firing sequence. Therefore, there must be a $\mu \in R(\mathscr{P})$ such that $\mu\left(p_{i}\right)=0$. Suppose, on the other hand, that there is a marking $\mu \in R(\mathscr{P})$ such that $\mu\left(p_{i}\right)=0$. Let $\sigma$ be such that $\mu_{0} \rightarrow \mu$. Then the firing sequence $\sigma t_{1}^{\prime} t_{2}^{\prime} t_{2}^{\prime} t_{2}^{\prime} \ldots$ is clearly $\infty$-fair.

Queille and Sifakis [30] have extended fairness in two other ways, namely, fair choice from states, and fair reachability of predicates. Applying these notions of fairness to Petri nets, we have that for a $P N \mathscr{P}=\left(P, T, \varphi, \mu_{0}\right)$, an infinite firing sequence $\sigma$ satisfies:

- fair choice from states (state-fair, for short) iff for any marking $\mu$ reached infinitely often by $\sigma$, every transition erabled at $\mu$ is executed infinitely often from $\mu$ in $\sigma$.

- fair reachability of predicates (pred-fair, for short) iff for any (finite or infinite) set of markings $M$, if there are infinitely many $i$ such that $\mu_{0} \underset{\sigma[i]}{\longrightarrow} \mu_{i} \stackrel{\sigma_{i}^{\prime}}{\rightarrow} \mu_{i}^{\prime} \in M$ for some $\sigma_{i}^{\prime}$, then there must be infinitely many $j$ such that $\mu_{0} \stackrel{\sigma[j]}{\longrightarrow} \mu_{j} \in M$.

At this time, we are unable to establish tight bounds for either NTP ${ }^{\text {state-farr }}$ or NTP pred-fair. NTPstate-fair seems to be related to the problem of finding a home state, that is, a marking that is reachable from any reachable marking (see [12], where a decision procedure was given to determine whether a given marking is a home state). Because the definition of pred-fair is quantified over all sets of markings, it appears to be a very difficult problem. The next three theorems give the bounds we are able to derive for these two problems.

Theorem 4.18. NTP ${ }^{\text {state-fair }}$ is decidable.

Proof. Let $\mathscr{P}$ be an arbitrary PN. We first determine whether $\mathscr{P}$ is bounded. If $\mathscr{P}$ is unbounded, there is an infinite firing sequence $\sigma$ which reaches each marking at most once. $\sigma$ is clearly state-fair. On the other hand, if $\sigma$ is bounded, we can construct the reachability graph. Then there is an infinite state-fair firing sequence iff the reachability graph contains a strongly connected component from which there is no exit.

We now define the following problems, which will be used in giving our lower bound for NTP state-fair. $^{\text {stir }}$

- BRP:

Instance: Given a bounded Petri net $\mathscr{P}$ and a marking $\mu$,

Question: Is $\mu \in R(\mathscr{P})$ ?

- BRP-S0:

Instance: Given a bounded Petri net $\mathscr{P}$ with a designated place $p$,

Question: Is there a $\mu \in R(\mathscr{P})$ such that $\mu(p)=0$ ?

The following lemma can be shown in a similar manner as Lemma 4.8. 
Lemma 4.19. $B R P-S O \equiv \equiv_{\text {PTIME }} B R P$.

Although it follows from $[31,23]$ that $B P \leqslant_{\text {PTIME }} B R P \leqslant_{\text {PTIME }} R P$, we do not know whether either of these reductions can be strengthened to an equivalence.

Theorem 4.20. BRP $\leqslant$ PTIME NTP State-fair. $^{\text {. }}$

Proof. We will use the fact that BRP-S0 $\equiv_{\text {PTIME }}$ BRP. I.et $\mathscr{P}=\left(P, T, \varphi, \mu_{0}\right)$ be an arbitrary bounded PN with a designated place $p_{i}$. We construct $\mathscr{P}^{\prime}$ as shown in Fig. 10. Suppose there is a $\mu \in R(\mathscr{P})$ such that $\mu\left(p_{i}\right)=0$. Let $\sigma$ be such that $\mu_{0} \rightarrow \mu$. Then the sequence $\sigma t_{1}^{\prime} t_{2}^{\prime} t_{2}^{\prime} t_{2}^{\prime} \ldots$ in $\mathscr{P}^{\prime}$ is clearly state-fair. Now suppose there is an infinite state-fair path $\sigma$ ia $\mathscr{P}^{\prime}$. Since $\mathscr{P}$ is bounded, $\mathscr{P}^{\prime}$ is clearly bounded. Since $t_{1}^{\prime}$ is enabled until it is fired, it must be fired in $\sigma$; otherwise, there would be some marking reached infinitely often by $\sigma$ in which $t_{1}^{\prime}$ is enabled. Since $t_{1}^{\prime}$ fires, $t_{3}^{\prime}$ cannot fire; hence, there must be a marking $\mu \in R(\mathscr{P})$ such that $\mu\left(p_{i}\right)=0$. Therefore, $B R P \leqslant_{\text {PTIME }} B R P-S 0 \leqslant$ PTIME $N T P^{\text {state-fair }}$.

Theorem 4.21. RP $\leqslant_{\text {PTIME }}$ NTP ${ }^{\text {pred-fair }}$.

Proof. We will use the fact that RP-S0 $\equiv_{\text {PTIME }}$ RP. Let $\mathscr{P}=\left(P, T, \varphi, \mu_{0}\right)$ be an arbitrary PN with a designated place $p_{i}$. We again construct $\mathscr{P}^{\prime}$ as shown in Fig. 10. Suppose there is a $\mu \in R(\mathscr{P})$ such that $\mu\left(p_{i}\right)=0$. Let $\sigma$ be such that $\mu_{0} g \vec{g} \mu$. We claim that $\sigma^{\prime}=\sigma t_{1}^{\prime} t_{2}^{\prime} t_{2}^{\prime} t_{2}^{\prime} \ldots$ is pred-fair. To see this, first note that after $t_{1}^{\prime}$ fires only one marking is reachable. Since this marking is reached infinitely often by $\sigma^{\prime}$, $\sigma^{\prime}$ must be pred-fair. Now suppose that there is an infinite pred-fair firing sequence $\sigma$ in $\mathscr{P}^{\prime}$. Let $M=\left\{\mu \mid \mu\left(p^{\prime}\right)=0\right.$ and $\left.\mu\left(p_{2}^{\prime}\right)=0\right\}$. Since no marking in $M$ can be reached in an infinite firing sequence, there must be some $i \in N$ such that $\mu_{0} \stackrel{\sigma[i]}{\longrightarrow} \mu^{\prime}$, such that no marking in $M$ is in $R\left(P, T, \varphi, \mu^{\prime}\right)$. Clearly, $t_{3}^{\prime}$ cannot occur in $\sigma[i]$, nor can it be enabled at $\mu^{\prime}$. Therefore, there must be some $\mu \in R(\mathscr{P})$ such that

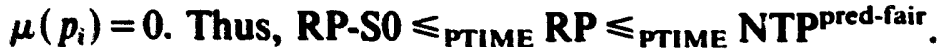

Finally, we examine equifairness as defined by Francez [13]. Given a Petri net $\mathscr{P}$ and an infinite firing sequence $\sigma, \sigma$ is said to be

- equifair iff there exist infinitely many $i$ such that all transitions occur the same number of times in $\sigma[i]$.

We will show this problem to be equivalent to the BP. In order to show this, we must introduce some terminology from [31] (see also [32]). Let $\mathscr{P}=\left(P, T, \varphi, \mu_{0}\right)$ be a PN. A generalized marking is a mapping $\mu: P \rightarrow Z$, where $Z$ is the set of integers. A generalized firing sequence from $\mu_{0}$ is any sequence of transitions. We then extend the notation $\mu\lrcorner \mu^{\prime}$ to generalized markings. An i-loop is a nonempty sequence $\sigma$ of transitions $\mu_{0} \stackrel{g}{\rightarrow} \mu_{0}$ such that there are $i$ places $p_{1}, \ldots, p_{i}$ such that for any $j \leqslant n$, where $n$ is the length of $\sigma, \mu_{0} \stackrel{\sigma[j]}{\longrightarrow} \mu_{j}$ and $\mu_{j}\left(p_{i}\right) \geqslant 0$ for $1 \leqslant i^{\prime} \leqslant i$. Using a strategy similar to that of Rackoff [31], we can show the following lemma. 


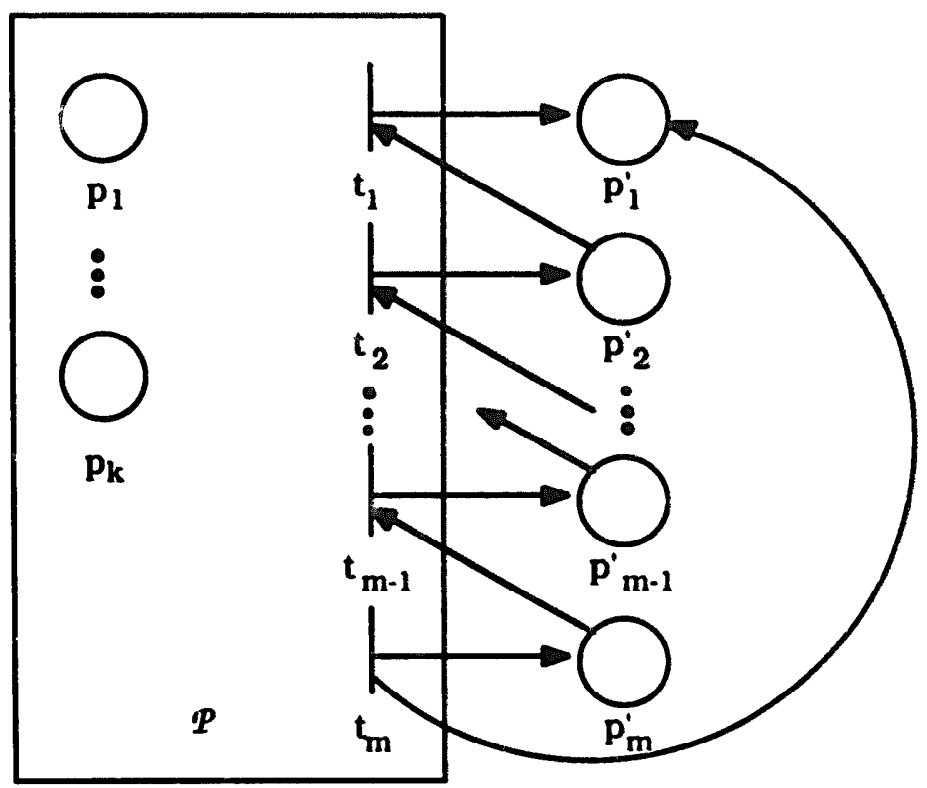

Fig. 11. $\mathscr{P}^{\prime}$ for equifairness.

Lemma 4.22. Given a $P N \mathscr{P}=\left(P, T, \varphi, \mu_{0}\right)$ and an $i \leqslant|P|$, we can decide in space $2^{\text {cnlogn }}$ for some constant $c$ whether there is an i-loop in $\mathscr{P}$.

We can now show the following.

Theorem 4.23. NTP ${ }^{\text {equifair }} \equiv_{\text {PTIME }}$ BP.

Proof. We first show NTPequifair $\leqslant$ ptime BP. Let $\mathscr{P}=\left(P, T, \varphi, \mu_{0}\right)$ be an arbitrary PN. We construct $\mathscr{P}^{\prime}$ as shown in Fig. 11. Ciearly, $\mathscr{P}^{\prime}$ has a $|P|$-loop iff $\mathscr{P}$ has an: infinite equifair firing sequence. Therefore, from Lemma 4.22 and [23], NTPequifair $\leqslant$ PTIME BP.

We now show BP $\leqslant_{\text {PTIME }}$ NTPequifair. We will use the fact that CP $\equiv_{\text {PTIME }}$ BP. Let $\mathscr{P}$ be an arbitrary $\mathrm{PN}$, and let $\mu$ be an arbitrary marking. We construct $\mathscr{P}^{\prime}$ as in Theorem 4.7. Clearly, if $\mathscr{P}^{\prime}$ has an infinite equifair path, there is a $\mu^{\prime} \in R(\mathscr{P})$ such that $\mu^{\prime} \geqslant \mu$. Suppose, conversely, that $\mu_{0} \stackrel{\sigma}{\rightarrow} \mu^{\prime} \geqslant \mu$. (Here, $\sigma$ is a firing sequence, as opposed to a generalized firing sequence.) Let $i$ be the maximum number of occurrences of any transition in $\sigma$. Clearly, $\sigma$ followed by $i$ occurrences of $t^{\prime}$ is a firing sequence in $\mathscr{P}^{\prime}$. This firing sequence can then be followed by enough occurrences of each transition so that each transition occurs exactly $i$ times. This can then be repeated infinitely many times. Therefore, $B P \leqslant_{\text {PTIME }} C P \leqslant_{r \text { rime }} N T P^{\text {equifair }}$.

\section{Conclusion}

We have exhibited a temporal logi , powerful enough to express certain fairness constraints, yet whose FMCP is equivalent to the reachability problem for PNs. 
Table 1

\begin{tabular}{|c|c|c|c|}
\hline NTP & Complexity & NTP & Complexity \\
\hline M1 & $\mathbf{R P}$ & imp & BP \\
\hline M1' & NL & just & $\geqslant \mathbf{R} \mathbf{P}$ \\
\hline M2 & $\mathbf{R P}$ & fair & $\Sigma_{1}^{1}$ \\
\hline M2' & RP & bd-fair & NPR \\
\hline 13 & RP & fdp- $\mathscr{T}$ & $\geqslant \mathbf{R P}$ \\
\hline $\mathrm{M}^{\prime}$ & $\mathbf{R P}$ & fdp- $T^{\mathrm{b}}$ & $\mathbf{R P}$ \\
\hline $\mathrm{Tl}$ & BP & fair- $\bar{J}$ & $\Sigma !$ \\
\hline $\mathrm{Tl}^{\prime}$ & BP & i-fair & $\therefore 1$ \\
\hline $\mathrm{T} 2$ & BP & $\infty$-fair & $\mathbf{R P}$ \\
\hline $\mathrm{T} 2^{\prime}$ & BP & state-fair & $\mathrm{D}, \geqslant \mathrm{BRP}$ \\
\hline $\mathrm{T} 3$ & BP & pred-fair & $\geqslant \mathbf{R} \mathbf{P}$ \\
\hline T3' & BP & equifair & BP \\
\hline
\end{tabular}

$a \geq B R P$, as hard as the BRP; $\geqslant R P$, as hard as the RP; NrR, decidable, bit not primitive recursive; $D$, decidable.

${ }^{b}$ fdp- $T$, fdp- $\mathscr{T}$ with $|\mathcal{T}|=1$.

This legic was instrumental in shio:ving seven fair nontermination problems to be equivalent to RP. In develcping this logic, we were able to answer a question left open in [16], namely, is there a decision procedure for $\mathscr{L}^{\infty}\left(Q^{\prime}\right)$ ? W'e were able to give a positive answer to this question and show that the problem is equivalent to reachability. One question that remains open is whether there is a decision procedure for $\tilde{L}\left(Q^{\prime}, \boldsymbol{G F}\right)$; i.e., wffs including predicates from $Q^{\prime}$ and the operators $\boldsymbol{G F}, \wedge, \vee$, and $\neg$, where $\neg$ is allowed only on predicates.

Table 1 summarizes the fairness results of this paper.

Most of the problems examined have been shown to be either equivalent to boundedness, equivalent to reachability, or $\Sigma_{1}^{1}$-complete. Two exceptions to this general rule are $\mathrm{NTP}^{\mathrm{MI}}$ and $\mathrm{NTP}^{\text {hd-fair. }}$. NTP ${ }^{\mathrm{MI}}$ can be decided in nondeterministic logspace because the entire allowable reachability se is explicitly given as input. Using the fact that bounded PNs can generate very large numbers, we were able to show that NTP ${ }^{\text {bd-fair }}$ is not primitive recursive. Aside from the fact that the precise complexity of RP is still unknown, the precise complexities of four of the fair nontermination problems we have examined remain open. Actually, Jančar [19] has rerently shown NTP ${ }^{\text {just }}$ and NTP ${ }^{\text {fdp. } . j}$ to be decidable. These two problems are particularly interesting because they are related to the open temporal logic question mentioned above; i.e., they are both expressible in $\tilde{\mathscr{L}}\left(Q^{\prime}, \boldsymbol{G F}\right)$. It will be interesting to see if Jančar's techniques can be extended to $\tilde{\mathscr{L}}\left(Q^{\prime}, \boldsymbol{G F}\right)$.

\section{Acknowledgment}

We would like to thank Prof. H. Carstensen for his suggestions and encouraging comments. In particular, we thank him for suggesting that model checking with 
respect to general PNs might be decidable for $\tilde{\mathscr{L}}\left(Q^{\prime}, F, X\right)$. We would also like to thank the referees for their helpful comments.

\section{Note added in proof}

After the final revision of this paper was completed, Jančar extended his work to show that $\tilde{\mathscr{L}}\left(Q^{\prime}, G F\right)$ is, in fact, decidable. All of Jančar's results on NTP ${ }^{\text {just }}$, $\mathrm{NTP}^{\mathrm{fdp}-\mathcal{T}}$, and $\tilde{L}\left(Q^{\prime}, G F\right)$ may be found in [19].

\section{References}

[1] K. Apt and D. Kozen, Limits for automatic verification of finite-state concurrent systems, Inform. Process. Let: 22 (1986) 307-310.

[2] K. Apt and E. Olderog, Proof rules and transformations dealing with fairness, Sci. Comput. Prog. 3 (1983) 65-100.

[3] K. Apt, A. Pnueli and J. Stavi, Fair termination revisited with delay, in: Proc. 2nd Conf. on Foundations of Software Technology and Theoretical Computer Science (FST-TCS) (1982) 146-170.

[4] H. Baker, Rabin's pronf of the undecidability of the reachability set inclusion problem of vector aćdition systems, Miln Pro e.t MAC, 1973, CSGM 79, Cambridge, MA, 1973.

[5] E. Best, Fairness and conspiracies, Inform. Process. Lett. 18 (1984) 215-220; addendum 19 (1984) 162.

[6] G. Brams, Reseaux de Petri: Théorie et Pratique-Tome 1: Théorie et Analyse (Masson, Paris, 1983).

[7] H. Carstensen, Decidability questions for fairness in Petri nets, in: Proc. 4th Symp. on Theoretical Aspects of Computer Science, Lecture Notes in Computer Science 247 (Springer, Berlin, 1987) 396-407.

[8] H. Carstensen and R. Valk, Infinite behaviour and fairness in Petri nets, in: Advances in Petri Nets 1984, Lecture Notes in Computer Science 188 (Springer, Berlin, 1985) 83-100.

[9] A. Chandra, Computable nondeterministic functions, in: Proc 19th IEEE Symp. on the Foundations of Computer Scicnce (1978) 127-131.

[10] E. Clarke and E. Emerson, Design and synthesis of synchronization skeletons using branching time temporal logic in: Workshop on Logics of Pregrams, Lecture Notes in Computer Science 131 (Springer, Berlin, 1981) 52-71.

[11] E. Emersor: and C. Lei, Modalities for model checking: Branching time logic strikes back, Sci. Comput. Prog. 8 (1987) 275-306.

[12] D. Escrig, A collection of algorithms to decide liveness and other related properties of a place transition system, Unpublished manuscript, Dpto. Informática y Automática, Fac. Ciencias Matemáticas, Universidad Complutense, 28040 Madrid, Spain.

[13] N. Francez, Fairness (Springer, Berlin, 1986).

[14] M. Hack, The equality problem for vector addition systems is undecidable, Theoret. Comput. Sci. 2 (1976) 77-95.

[15] D. Harel, Effective transformations on infinite trees, with applications to high undecidability, dominoes, and fairness, J. ACM 33 (1986) 224-248.

[16] R. Howell and L. Rosier, Problems concerning fairness and temporal logic for conflict-free Petri nets, Theoret. Comput. Sci. 64 (1989) 305-329.

[17] R. Howell, L. Rosier, D. Huynh and H. Yen, Some complexity bounds for problems concerning finite and 2-dimensional vector addition systems with states, Theoret. Comput. Sci. 46 ( 1986) i07-140.

[18] N. Immerman, Nondeterministic space is closed under complementation, SIAM J. Comput. 17 (1988) 935-938.

[19] P. Jančar, Decidability of a temporal logic problem for Petri nets, Theoret. Comput. Sci. 74 (1990) 71-93

[20] R. Kosaraju, Decidar:lity of reachability in vector addition systems, in: Proc. 14th innu. ACM Symp. on Theory of Co:nputing (1982) 267-280. 
[21] L. Landweber, Desision problems for $\omega$-automata, Math. Systems Theory 3 (1969) 376-384.

[22] D. Lehman, A. Pnueli and J. Stavi, Impartiality, justice, and fairness: the ethics of concurrent termination, in: Proc. 8th Internat. Colloquium on Automata, Languages, and Programming, Lecture Notes in Computer Science 115 (Springer, Berlin, 1981) 264-277.

[23] R. Lipton, The Reachability Problem Requires Exponential Space, Technical Report 62, Yale University, Dept. of CS., 1976.

[24] Z. Manna and A. Pnueli, The modal logic of programs, in: Proc, 6th Internat. Colloquitum on Automata, Languages, and Programming, Lecture Notes in Cumputer Science 71 (Springer, Berlin, 1979) 385-410.

[25] E. Mayr, An algorithm for the general Petri net reachability problem, SIAM J. Comput. 13(3) (1984) 441-460; A preliminary version of this paper was presented at the 13th Annu. Symp. on Theory of Computing (1981).

[26] H. Müller, Weak Petri net computers for Ackermann functions, Elekt.on. Informationsverarb. Kybernet. 21 (1985) 236-244.

[27] E. Olderog and K. Apt, Transformations realizing fairness assumptions for parallel programs, in: Proc. STACS '84, Lecture Notes in Computer Science 166 (Springer, Berlin, 1984) 20-42.

[28] J. Peterson, Fetri Net Theory and the Modeling of Systems (Prentice Hall, Englewood Cliffs, NJ, 1981).

[29] A. Pnueli, The temporal logic of programs, in: Proc. 19th Annu. Symp. on Foundations of Computer Science (1977).

[30] J. Queille and J. Sifakis, Fairness and related properties in transition systems-a temporal logic to deal with fairness, Acta Inform. 19 (1983) 195-220.

[31] C. Rackoff, The covering and boundedness problems for vector addition systems, Theoret. Comput. Sci. 6 (1978) 223-231.

[32] L. Rosier and $H$. Yen, A multiparameter analysis of the boundedness problem for vector addition systems, J. Comput. System Sci. 32(i) (1986) 105-135.

[33] A. Sistla and E. Clarke, The complexity of propositional linear temporal logic, J. ACM 32 (1985) 733-749.

[34] A. Sistla and S. German, Reasoning with many processes, in: Proc. IEEE Symp. on Logic in Computer Science (IEEE, Ithaca, NY, 1987) 138-152.

[35] I. Suzuki, Fundamental properties and applications of temporal Petri nets, in: Proc. 19th Annu. Conf. on Injormation Sciences and Systems (1985) 641-646.

[36] R. Szelepcsényi, The method of forced enumeration for nondeterministic automata, Acta Inform. 26 (1988) 279-284.

[37] R. Tarjan, Depth first search and linear graph algorithms, SIAM J. Comput. 1 (1972) 146-160.

[38] R. Valk and $M$. Jantzen, The residue of vector sets with applications to decidability problems in Petri nets, Acta Inform. 21 (1985) 643-674. 